\title{
Adapting a General-Purpose Social Robot for Paediatric Rehabilitation through In Situ Design
}

\author{
FELIP MARTÍ CARRILLO, Swinburne University of Technology and Data61, CSIRO, Australia \\ JOANNA BUTCHART, Royal Children's Hospital and Murdoch Children's Research Institute, Australia \\ SARAH KNIGHT, Murdoch Children's Research Institute and Royal Children's Hospital, Australia \\ ADAM SCHEINBERG, Royal Children's Hospital and Murdoch Children's Research Institute, Australia \\ LISA WISE, LEON STERLING, and CHRIS MCCARTHY, Swinburne University of Technology, \\ Australia
}

\begin{abstract}
Socially assistive robots (SARs) offer great promise for improving outcomes in paediatric rehabilitation. However, the design of software and interactive capabilities for SARs must be carefully considered in the context of their intended clinical use. While previous work has explored specific roles and functionalities to support paediatric rehabilitation, few have considered the design of such capabilities in the context of ongoing clinical deployment. In this article, we present a two-phase in situ design process for SARs in health care, emphasising stakeholder engagement and on-site development. We explore this in the context of developing the humanoid social robot NAO as a socially assistive rehabilitation aid for children with cerebral palsy. We present and evaluate our design process, outcomes achieved, and preliminary results from ongoing clinical testing with 9 patients and 5 therapists over 14 sessions. We argue that our in situ design methodology has been central to the rapid and successful deployment of our system.
\end{abstract}

CCS Concepts: • Human-centered computing $\rightarrow$ Field studies; $\bullet$ Computer systems organization $\rightarrow$ Robotics; • Applied computing $\rightarrow$ Health care information systems; $\bullet$ Social and professional topics $\rightarrow$ Children;

Additional Key Words and Phrases: In situ design, socially assistive robots, rehabilitation, health care

ACM Reference format:

Felip Martí Carrillo, Joanna Butchart, Sarah Knight, Adam Scheinberg, Lisa Wise, Leon Sterling, and Chris McCarthy. 2018. Adapting a General-Purpose Social Robot for Paediatric Rehabilitation through In Situ Design. ACM Trans. Hum.-Robot Interact. 7, 1, Article 12 (May 2018), 30 pages.

https://doi.org/10.1145/3203304

Project funding provided by the Transport Accident Commission (TAC) grant for Phase 1; Data61 and the CSIRO student scholarship provided funding for Phase 2 .

Authors' addresses: F. Martí Carrillo, Swinburne University of Technology, H39 PO Box 218, Hawthorn, VIC 3122, Australia; email: fmarti@swin.edu.au; J. Butchart, Royal Children’s Hospital, Victorian Paediatric Rehabilitation Service, 50 Flemington Road, Parkville, VIC 3052; email: jo.butchart@rch.org.au; S. Knight, Royal Children’s Hospital, Victorian Paediatric Rehabilitation Service, 50 Flemington Road, Parkville, VIC 3052; email: sarah.knight@mcri.edu.au; A. Scheinberg, Royal Children's Hospital, Victorian Paediatric Rehabilitation Service, 50 Flemington Road, Parkville, VIC 3052; email: adam.scheinberg@rch.org.au; L. Wise, Swinburne University of Technology, H24 PO Box 218, Hawthorn, VIC 3122, Australia; email: lwise@swin.edu.au; L. Sterling, Swinburne University of Technology, H80 PO Box 218, Hawthorn, VIC 3122, Australia; email: lsterling@swin.edu.au; C. McCarthy, Swinburne University of Technology, H39 PO Box 218, Hawthorn, VIC 3122, Australia; email: cdmccarthy@swin.edu.au.

Permission to make digital or hard copies of all or part of this work for personal or classroom use is granted without fee provided that copies are not made or distributed for profit or commercial advantage and that copies bear this notice and the full citation on the first page. Copyrights for components of this work owned by others than ACM must be honored. Abstracting with credit is permitted. To copy otherwise, or republish, to post on servers or to redistribute to lists, requires prior specific permission and/or a fee. Request permissions from permissions@acm.org.

(C) 2018 ACM 2573-9522/2018/05-ART12 \$15.00

https://doi.org/10.1145/3203304

ACM Transactions on Human-Robot Interaction, Vol. 7, No. 1, Article 12. Publication date: May 2018. 


\section{INTRODUCTION}

Rehabilitation outcomes rely critically on patients adhering to a prescribed set of rehabilitation exercises [38]. When those patients are children, maintaining compliance and focus while performing what can often be tiring, uncomfortable, and repetitive exercise programs presents a significant challenge [30,34]. While therapists and caregivers are well equipped with skills and experience to maintain a child's motivation, this takes considerable time and resources [24]. Therapists are not always able to attend each prescribed exercise session and, even when present, results are not always positive.

Socially assistive robots (SARs) are increasingly being considered to support a range of health care delivery needs. SARs provide assistance primarily through social interaction and engagement [8], that is, children suffering form serious illness [1]. SARs have shown promising results for improving mood, reducing stress, and encouraging communication for children on the autism spectrum [35], in rehabilitation [4, 37], for encouraging exercise in older adults [7], and in poststroke rehabilitation [44].

Paediatric rehabilitation presents an ideal context for the application of SARs. Previous work suggests that SARs may provide therapeutic benefits for patients through increased focus and compliance [9, 17]. However, no formal clinical evaluation of the therapeutic benefits of SARs for rehabilitation currently exists. This requires development beyond proof-of-concept, with clear clinical-use cases identified. While previous work has explored specific roles and functionalities to support paediatric rehabilitation (e.g., $[2,4,20,37]$ ) few have considered the design of such capabilities in the context of ongoing clinical deployment. Addressing this gap is critical to understanding the clinical context that SARs must operate in and for establishing the long-term legitimacy of SARs as effective and usable therapeutic aids with therapists and caregivers.

We are developing software to adapt the humanoid robot NAO as a therapeutic aid for paediatric rehabilitation and evaluating its effectiveness. In partnership with a busy paediatric rehabilitation clinic of The Royal Children's Hospital, Melbourne, Australia, we are developing a range of interactive and demonstrative behaviours for NAO to enhance patient compliance, motivation, and emotional well-being during therapy sessions. We aim to deploy NAO robots as both a therapist's assistant during sessions and as a proxy for therapists when they are unable to attend (e.g., onward after-hours or at home). The near-term goal is thus to autonomously support independent exercise programs on the ward, before then extending the system's use to supporting prescribed rehabilitation programs at home. To this end, we are determining roles and developing robust interactive capabilities that allow NAO to guide patients through complete exercise sessions without engineer monitoring (or Wizard-of-Oz control) or additional hardware (e.g., external sensors).

In this article, we report on 23 months of progress designing and developing software for NAO as a therapeutic aid for paediatric rehabilitation. Focused on the needs of large-scale clinical deployment, we outline key requirements for an SAR operating as a stand-alone therapeutic aid for ongoing use in a clinical setting. We present a two-phase in situ design process, including both exploration of roles and requirements, from which a base-level stand-alone prototype system has been derived. To our knowledge, this is the first design of an SAR for rehabilitation that explicitly incorporates patients, caregivers, and therapists in the design process, and is focussed on the design of roles and capabilities for ongoing use in a clinical setting. Our prototype system is now deployed in weekly therapy sessions, predominantly leading patients with cerebral palsy $(\mathrm{CP})$ through prescribed exercise programs of up to 30 minutes without engineer intervention.

The article is structured as follows. Section 2 gives background and an overview of previous work. Section 3 outlines our in situ design methodology, listing derived roles and requirements for the system from Phase 1 of this process. Sections 4 and 5 provide a technical overview of the 
current system deployed in Phase 2 development and key design choices and considerations. We present our clinical testing setup, a discussion of preliminary Phase 2 results, and feedback in Section 6. Our conclusions are presented in Section 7.

\section{BACKGROUND AND RELATED WORK}

\subsection{Socially Assistive Robots in Paediatric Rehabilitation}

A number of groups have considered SARs for rehabilitation, focussing primarily on technical developments and evaluations of proof-of-concept systems. "Ursus" [37] is a combination of a low-cost robot and an augmented reality device to assist upper limb rehabilitation exercises for children with CP. The system was evaluated in single sessions with six patients, with feedback suggesting SAR-enhanced enjoyment, and that the system had a positive impact on rehabilitation sessions. "Therapist" [4], the evolution of the "Ursus" robot platform, provides a virtual reality video game and exercise demonstrations for upper-limb exercises. A thorough evaluation of the system's cognitive framework (e.g., speech/emotion recognition, human detection, and so on) is provided from both lab-based and in-the-field experiments. Exercise demonstration and robot mirroring is also proposed by Fridin et al. [9] to assist groups of paediatric patients and Malik et al. [20], who implement three different exercise demonstrations (Sit to Stand, Balancing, and Ball kicking). "MARKO" [2], a robot sitting on a horse-like mobile platform, is designed to assist rehabilitation for patients with $\mathrm{CP}$ in gross motor skill exercises, fine motor skills, and speech exercises.

While previous systems have been tested with patients, no existing SAR has been deployed as part of the ongoing rehabilitation program of paediatric patients.

\subsection{In situ Design and Evaluation in the Wild}

Human-robot interaction (HRI) and human-computer interaction researchers have previously reported issues in the extrapolation of lab-based evaluations into real-world contexts. In the hospital context, Multu and Forlizzi [27] describe the rejection of deployed autonomous delivery robots by hospital staff owing to interruptions and distractions inflicted on them when performing higherpriority tasks. Such issues have promoted the use of in situ design and in the wild evaluation methodologies in which new technologies are designed and evaluated in place and under the conditions of their intended use [36].

Museums and public spaces have been a popular target of in-the-wild HRI design and evaluation $[3,40]$ as well as in the home. Kidd and Breazeal [15] report on the in situ design and evaluation of a weight loss coach robot, benchmarking it against a stand-alone computer and a traditional paper log. They show a two-fold increase in exercise time for participants using the robot compared to those using the aids. Hüttenrauch et al. [13] study participant interaction patterns with a mobile robot in a home-guided tour. More recently, Pripfl et al. [32] report on the results of an in-thewild evaluation of a service robot deployed in the homes of 18 elderly participants. Their findings highlight issues with both technical performance of the system and participant perceptions of the robot as a toy rather than an aid.

Šabanović et al. [41] report on the in situ design and development of a robot to manage break times in an office environment. They note benefits for identifying contextual issues impacting robot use and for including users in the design process even when evaluating with incomplete and nonrobust prototypes. CERO [12] was used to assist in the transport of objects in an office environment for partially motion-impaired users over a 3-month study. The in situ evaluation of the prototype identified important factors not considered previously, such as physical space limitations and bystander engagement. An in situ HRI study by Michalowski et al. [25] examined social 
engagement with two social robots in a conference setting. This evaluation identified flawed design assumptions, leading to new ideas and improvements in the robot's interaction effectiveness.

The in situ design of SARs in health settings is less common, though examples of evaluation during deployment exist. Studies using the seal robot PARO, for example, have shown benefits for improving mood, reducing stress, and encouraging social engagement for residents in an eldercare facility. Such studies have performed evaluations over 5 weeks [43], 4 months [16], and 1 year [42]. In situ studies have also evaluated PARO as a therapeutic aid for people with dementia [5, 10, 26]. Most closely aligned to our application, Plaisant et al. [29] employed a participatory design approach in the design of an SAR prototype to enhance rehabilitation outcomes with children. They iteratively evaluated their prototypes during the design sessions with their intended final users. However, unlike our approach, they did not deploy the SAR to lead sessions or as part of the ongoing care delivery.

Our contributions differ from previous work in the following distinct ways. First, we focus specifically on the design of an SAR for ongoing therapeutic use by a therapist or caregiver and for leading entire therapy sessions with children. Moreover, we adapt and evaluate a general-purpose social robot (NAO) as a stand-alone system, outlining design decisions and requirement compromises to achieve this. Finally, we outline and evaluate our design process for SARs in rehabilitation, noting specific design outcomes resulting from our in situ design and evaluation and the explicit inclusion of stakeholders in this design process.

\section{DESIGN PROCESS}

We have engaged in a two-phase in situ design process, incorporating both exploratory and iterative prototyping and frequent engagement with key stakeholders. In this section, we describe the project context, stakeholders, and the implementation of these two phases of development.

\subsection{Project Setting}

The proposed SAR system is being developed in close partnership with a busy paediatric rehabilitation clinic in a city-based children's hospital. The rehabilitation clinic consists of 25 full-time equivalent clinical staff servicing, on average, 180 inpatients annually as well as several thousand outpatient sessions. Patients seen at the clinic range from those recovering from physical injury and illness to those being treated for specific chronic disabilities. Inpatients generally undergo intensive rehabilitation programs requiring multiple sessions of rehabilitation per day. While some sessions are supervised by physiotherapist staff, others may be facilitated by on-ward nursing staff or the patient's parent. A particularly prominent patient group consists of children with CP. In many cases, orthopaedic surgery is required to correct secondary musculoskeletal problems that impact gait and function. Such patients typically undergo up to three rehabilitation sessions per day over a 2- to 3-week period [39].

\subsection{Stakeholders}

We identified the following four groups as key stakeholders in the development of the SAR for rehabilitation.

Patients: The primary beneficiaries of the SAR through potentially increased motivation and sustained emotional well-being, faster recovery time and improved rehabilitation outcomes. They are chief determinants of the SAR's interaction design.

Therapists/Health Care Providers: Primary users of the system, with use cases spanning both in-session use as well as preconfiguration for sessions without their direct 


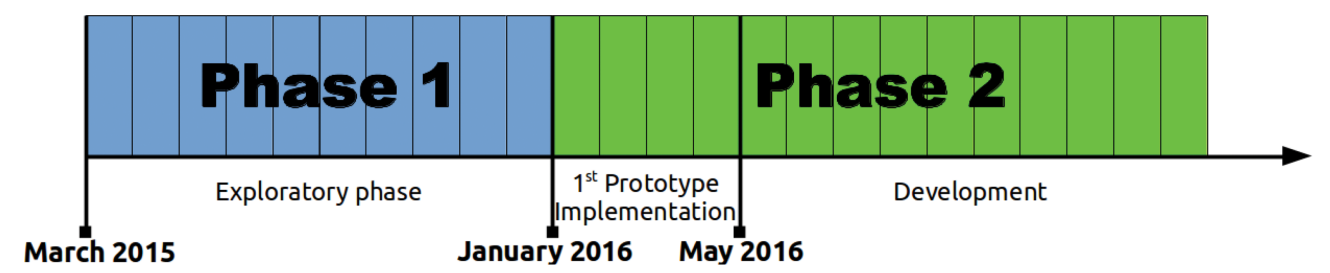

Fig. 1. Timeline of the project up to the current stage.

supervision. They are determinants of the SAR's therapeutic assistance, correctness (e.g., exercise demonstrations), usability, integration, and fitness for purpose.

Parents/Guardians: Holders of primary duty of care for patients, they are often present during therapy sessions and tasked with ensuring that rehabilitation exercises are performed outside of formal therapy sessions (e.g., on-ward, after-hours). They are thus targeted endusers of the system and determinants of the system's usability and fitness for purpose.

Technology Developers: They engage with all other stakeholders to determine the SAR system requirements, design and implement interactive behaviours, and operate the SAR during development and testing. They gather feedback from other stakeholders, assessing the system's technical performance and the feasibility of identified roles and requirements.

\subsection{Design and Development}

Our design approach consists of two phases. The first, an exploratory phase to elicit basic requirements, ran for 10 months: between March 2015 and January 2016. The second phase, involving the iterative development and in situ evaluation of a first prototype implementation, began in March 2016 and is ongoing. Through these design phases, a prototype for formal clinical trials is being targeted. Figure 1 shows the timeline of development to date. We describe both phases below.

\subsection{Phase 1: Exploration}

The initial phase of the SAR's design, previously described in [23], prioritised two key activities: regular and frequent (weekly) stakeholder engagement and rapid prototyping and mock-ups (via Wizard-of-Oz control) of proposed roles and capabilities. Both activities were conducted primarily on-site in the context of the SAR's intended deployment.

A regular weekly pattern of visits to the clinic was established in the early weeks of the phase. Each Tuesday morning attending research team members (typically two) set up NAO in a publicly visible and accessible location, close to consultation rooms with high visibility to patients, their families, and therapists. This facilitated regular, albeit brief, discussions with therapists and parents at the beginning. Patient interactions were initially also brief, unstructured, and intermittent, typically occurring during their time waiting for a consultation with therapists. The use of Wizardof-Oz control via a laptop with wireless link to $\mathrm{NAO}$ allowed the SAR to meet the immediate needs of particular interactions.

Early engagement suggested how to overcome the technology limits and foster effective engagement with patients. It facilitated development of core exercise demonstrations. Therapists were actively engaged in this process, initially through requests to critique NAO's execution of exercises, and also invited to physically manipulate the robot's limbs to both correct and explore the physical capabilities and limitations of the system. 
In the second half of the phase, therapist engagement evolved into a cycle of iterative development in which a therapist directly programmed specific exercises by positioning the robot into key poses from which robot joint positions were immediately recorded and time sequenced. New exercises were rapidly developed via this process on-site, with refinements made between clinic visits. During this second half of Phase 1, observations determined specific roles (outlined in Section 3.6) based on the robot's capabilities and the derivation of requirements for an SAR (Section 3.7) for ongoing clinical use.

Patient engagement also progressed from nonspecific patient interactions driven primarily by general interest and the novelty of the robot in the waiting area to the active inclusion of NAO in therapist-selected patient sessions. Prebuilt exercise demonstrations were sequenced in accordance with therapist specifications and trialled in sessions with technical support. Early scripting of SAR behaviours was done using the vendor-supplied graphical development environment, Choreographe. This visual programming environment, while limiting in some technical respects owing to its highly abstracted block-style programming, allowed different technical developers to interchangeably operate NAO without requiring specialised knowledge of underlying system complexities, thereby increasing the pool of developers who could assist in this exploratory phase. This supported the maintaining of regular weekly visits throughout Phase 1 and diversified interactions between developers and all nontechnical stakeholders.

\subsection{Phase 2: Development}

Phase 2 is ongoing, prioritising the in situ iterative development and evaluation of a stand-alone prototype in preparation for formal clinical evaluation. As such, focus has been placed on the realisation of a minimum viable SAR based on the roles determined in Phase 1 and the identified key requirements in both phases for an SAR in rehabilitation [21].

Regular weekly patient sessions with NAO have been scheduled in which Wizard-of-Oz control and engineering support has been removed from the SAR's operation, thus focusing on the needs of ongoing stand-alone operation in a clinical setting. Phase 2 aims to develop the system to be under the sole operation of therapists, parents, and/or other caregivers.

Phase 1 established CP as a well-suited initial target for clinical evaluation. Phase 2 has thus focussed on a system capable of leading sessions for patients with $\mathrm{CP}$ undergoing postoperative rehabilitation. Exercise capabilities predominantly target lower-limb strengthening in accordance with the typical prescribed program of rehabilitation for this patient group.

Patients, therapists, and parents not involved in Phase 1 have been formally recruited and consented to participate in this phase of the study. Data is gathered via questionnaires with all stakeholders at the completion of each session, along with observation notes recorded during each session (detailed in Section 6.2). Attending researchers have observed from an adjacent room with a one-way mirror. We discuss the details of clinical sessions in Section 6.

\subsection{Derived Roles}

Therapist consultation and observation during Phase 1 determined four specific roles encompassing the base-level capabilities that the SAR must provide to serve as an effective therapeutic aid in rehabilitation sessions.

Demonstrator: At the beginning of each exercise set, the SAR performs the exercise in front of the child. The SAR also provides verbal instructions to emphasise important aspects of the exercise. 
Motivator: The SAR provides verbal encouragement at the beginning of each session as well as before and during each prescribed exercise. Enticements such as entertainment through music, dancing, and joke telling are also offered upon completion of exercise sets.

Companion: The SAR delivers personalised introductory statements at the beginning of the session to build rapport and establish itself as a joint participant in the session. As the child performs each exercise set, the SAR joins in and delivers empathetic and encouraging statements acknowledging the child's progress.

Coach: The SAR guides the patient through the prescribed session by scheduling and coordinating the execution of the above roles to deliver a complete session of therapy. The system paces the delivery in accordance with the patient and therapist/caregiver responses.

\subsection{Derived Requirements}

To support the above roles, Phase 1 identified the following system requirements.

3.7.1 Configurability. Therapists and technology developers in Phase 1 both identified the need for configurability of the system to realise a stand-alone SAR for rehabilitation. Early feedback from therapists requested a system based on current practise in which session schedules are produced by selecting activities from a list. Configuration thus needs to allow preselection of exercises to perform, number of repetitions, speed of execution, entertainment modules, as well as personalisation of the session with the patient.

3.7.2 Stability. Therapists and technology developers jointly determined that exercise demonstrations and general SAR actions must operate with a high degree of certainty in order to minimise session interruption and distraction. In the context of an off-the-shelf, general-purpose social robot, physical characteristics impacting this are not modifiable and thus must be carefully managed within the programmed movements of the system.

3.7.3 Adaptability. To ensure that therapeutic assistance is aligned with the patient's needs, the SAR should be adaptable to the presenting condition of the patient during care delivery. It was observed in Phase 1 that therapists prescribe exercises before a session but assess and adjust activities during the session. Therapists noted that an effective SAR for rehabilitation should provide mechanisms for dynamic adjustment of activity settings, including number of repetitions, speed, and sequence order. Verbal instructions must adjust accordingly.

3.7.4 Interaction. Observations in Phase 1 indicated a general desire of patients to interact with the robot, which should be facilitated often. Basic interaction with the SAR should always be supported for therapists/caregivers and patients throughout the session. Challenges observed with speech recognition during Phase 1 made clear that interaction should be multimodal (e.g., verbal, tactile, etc.) to cater to varying patient needs. This will support adaptability, responsiveness, and maintain patient engagement.

3.7.5 Integration. Previous work (e.g., Mutlu and Forlizzi [27]) and Phase 1 observations highlighted the need to ensure that setup and use of the SAR was well integrated with existing clinical practise and the general operating conditions of a busy hospital-based rehabilitation clinic. Therapists and technology developers together determined that the SAR must be easily set up by therapists and caregivers, must be portable and transportable by a single person, and operable by caregivers with minimal training requirements.

3.7.6 Responsiveness. Observations by technology developers in Phase 1 and early Phase 2 sessions indicated that a lack of responsiveness to unprompted verbal statements from patients may 


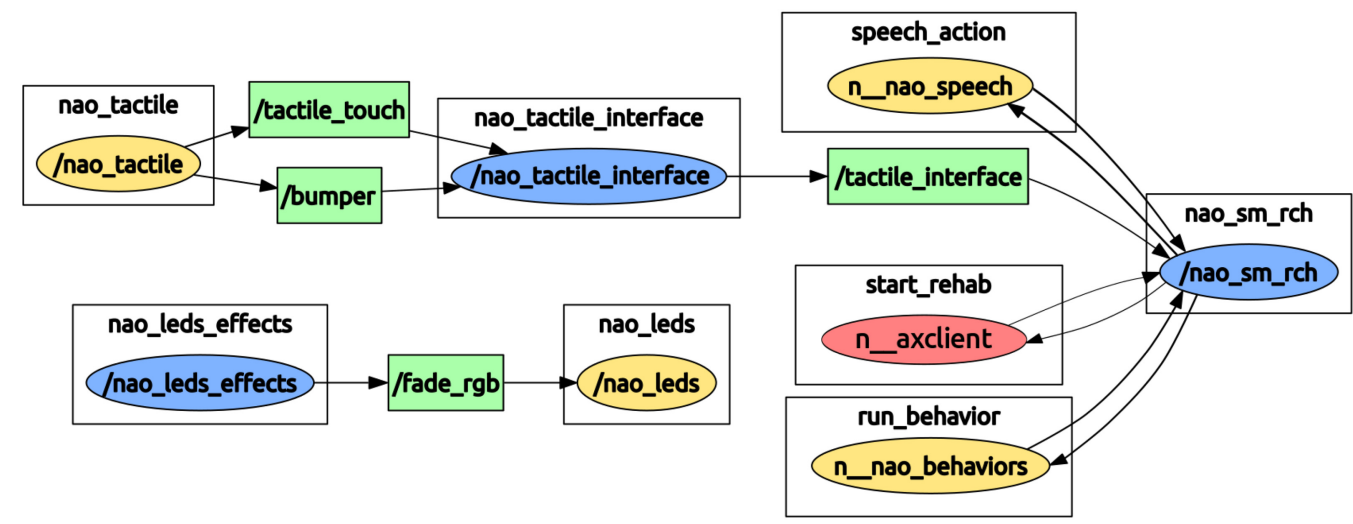

Fig. 2. ROS modules simplified for the SAR. In blue: ROS nodes implemented for the prototype. In yellow: ROS nodes that connect the ROS with NAO's server. In green: the ROS communication topics. In red: the graphical actionlib client to start the session from a remote computer.

diminish the perceived authenticity of the SAR's role as a companion. Observations also highlighted that the implementation of responses should also incorporate awareness of the patient's mood and progress to support the SAR's motivator role.

3.7.7 Stand-alone. Therapists and technology developers jointly agreed that the system should be operable without engineering support, Wizard-of-Oz control, or additional hardware to meet the needs of flexible and unhindered ongoing use. SAR activities requiring human assistance should also be minimised to ensure that caregiver focus remains primarily on the patient. Therapists also expressed a strong desire to have the SAR present and ready to use at the hospital at all times.

3.7.8 Robustness and Endurance. To meet the needs of leading rehabilitation sessions, therapists and technology developers determined that the system needs to operate continuously and for a minimum of 30 minutes without engineer intervention. To support the stand-alone requirement, unforeseen interruptions such as falls, slippage, or unintended/incorrect user interactions should also be recoverable from, either automatically or through a clearly understood set of instructions for the therapist and/or caregiver to follow.

In Sections 4 and 5, we outline the technical implementation and key design decisions to maximise the realisation of these baseline roles and requirements.

\section{SAR PROTOTYPE IMPLEMENTATION}

\subsection{Software Modules}

Our prototype software for the NAO robot platform utilises the Robot Operating System (ROS), an open-source robotics framework. The ROS was chosen on the basis of its extensibility and strong support for simplified communication between different tools and devices in a robotic system [33].

Figure 2 shows some of the basic modules of the NAO robot for ROS and the three modules implemented in our system. We briefly describe each below.

4.1.1 nao_sm_rch. is the main module of the system, incorporating all rehabilitation activity scenarios, including speech, lower-body exercises, games, and dances. We implement this as a finite state machine initiating specific scenarios via connections to other nodes of the system. 
Services such as run_behavior or speech_action are called from this node in order to execute a predefined movement or to make the robot speak. To assist data collection, the module also maintains a logfile tracking all the exercises executed, timing data, and user inputs.

4.1.2 nao_tactile_interface. This interface is implemented as an ROS service to capture and detect inputs to the system, such as from touch sensors and bumpers using the nao_tactile library. It detects single, double, and long button clicks, allowing numerous different responses to be invoked.

4.1.3 nao_leds_effects. This provides visual prompts and conveys the system state. We have configured this service using the ROS NAO library nao_leds with 5 different LED effects that are activated to cue the need for the robot's head to be tapped in order to continue the session or to indicate that a session configuration file is being loaded.

4.1.4 Other Nodes. Figure 2 shows other ROS libraries that we are using, such as nao_leds, nao_tactile, run_behavior, and speech_action. The robot is configured and started using the start_rehab action library.

\subsection{Activity Scenarios}

Our current prototype for Phase 2 trials implements 16 different activity scenarios to support the roles outlined in Section 3.6. Activity scenarios are all the rehabilitation exercises $(\mathrm{N}=13)$, plus an introductory speech delivery, a toy relay game, and entertainment routines. In the introductory speech, the robot introduces itself to the patient or greets a patient it has previously interacted with. In addition to statements explaining what is planned for the session, the scenario includes jokes and preprogrammed dialogue to foster rapport building. Several introductory speeches can be rotated to reduce repetition over multiple sessions.

Sessions consist of multiple exercises, each involving several sets and repetitions. Adjustments to exercise speed, if requested during the session, can be changed by the caregiver using the Tactile Interface, explained in more detail in Section 5.7. For each exercise, the SAR presents a demonstration while explaining key features of the exercise. The patient is then invited to join the SAR in completing a set with it. During exercise execution, the SAR provides encouraging and therapistselected reminders about key aspects of each exercise (Section 5.5.1). At the completion of each set, the SAR requests that the patient (or caregiver) tap its head to continue. The SAR asks for help when human assistance is required to set up a particular activity (Section 5.8).

The current Phase 2 prototype supports 13 different rehabilitation exercises: a sit-to-stand exercise (Figure 3) and 12 executable from a lying down position (Figure 5). These exercises represent core lower-body exercises typically prescribed in the rehabilitation program of patients with $\mathrm{CP}$. Exercises have been programmed with the help of physiotherapists through manual positioning of the unstiffened robot to capture key postures and the temporal sequence of transitions for each exercise [23]. This is supported using the vendor-supplied development environment, Choreographe [31].

Figure 4 depicts an activity scenario in which the robot guides patients through a so-called toy-relay game. In this scenario, the robot asks the patient to fetch named toys on the other side of the room. The activity encourages patients to walk while the robot provides instructions and motivational statements.

A final supported activity scenario provides a farewell, rewarding the patient's efforts at the end of the session with a dance. Dance options include one programmed entirely by a physiotherapist on the research team. 


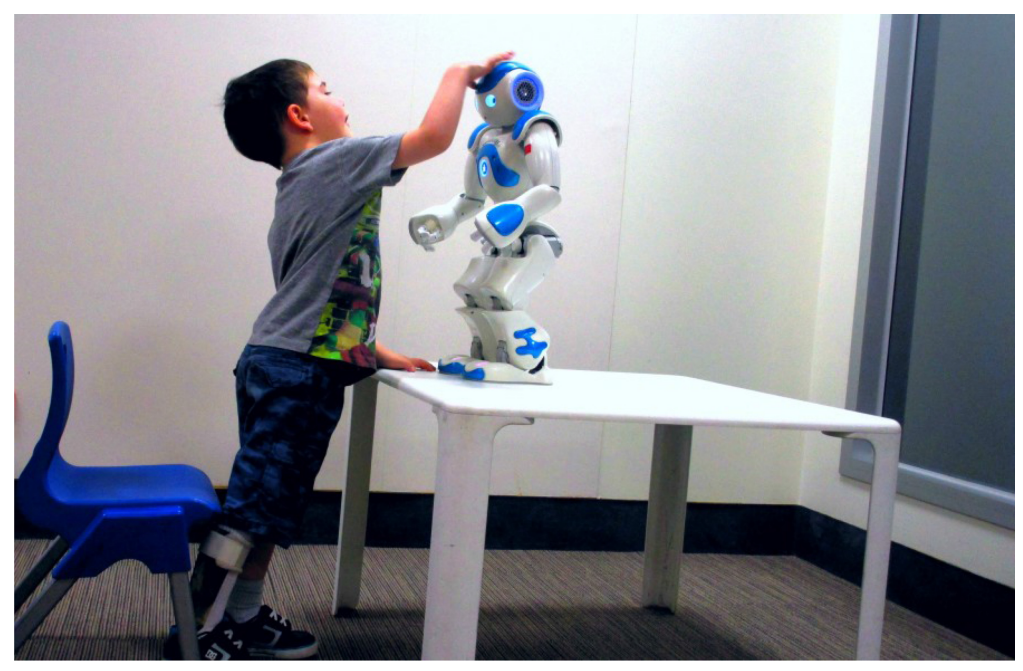

Fig. 3. Sit-to-Stand exercise: The patient taps the robot's head to initiate the robot's stand up sit down actions while the child follows. [Guardian consent provided]

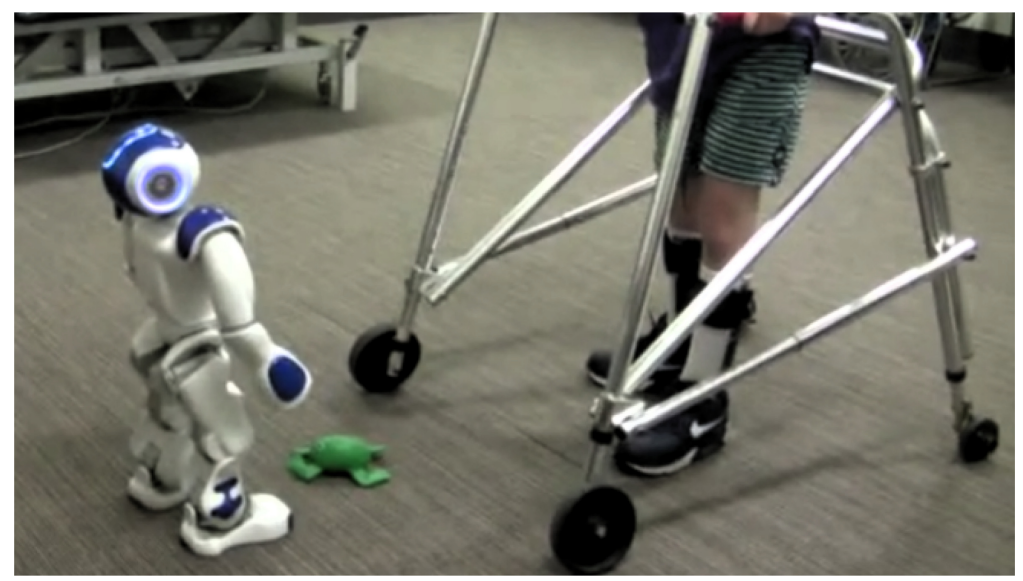

Fig. 4. NAO leads a patient with CP through the toy relay game during a therapy session. [Guardian consent provided]

\section{DESIGN DECISIONS}

The current Phase 2 prototype provides a baseline system enabling NAO to serve as an SAR for rehabilitation. Design requirements outlined in Section 3 have been carefully considered in the context of ensuring a reliable system for ongoing iterative development. In this section, we discuss specific design choices, compromises, and considerations that have been made to meet this objective.

\subsection{Activity Configuration Interface}

Phase 1 required program code to be explicitly written for each session to meet the needs of each individual patient. However, to fulfill both configurability and stand-alone requirements, all activity scenarios in the Phase 2 prototype (outlined in Section 4.2) are selectable and configurable via 
(a) Bridge

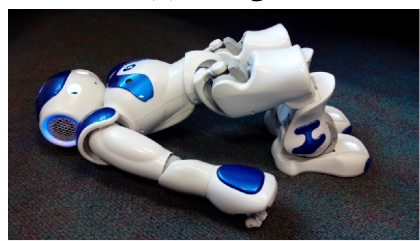

(d) Single Bridge

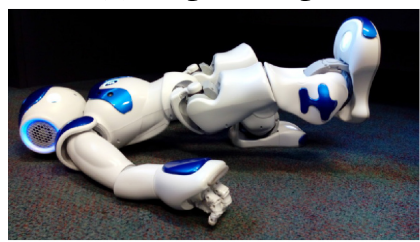

(g) Hip Knee Flexion Sliding

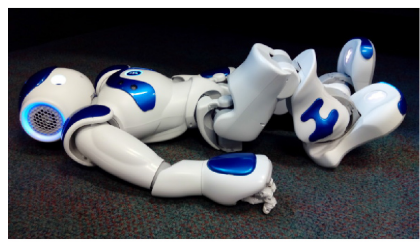

(j) Leg Raises

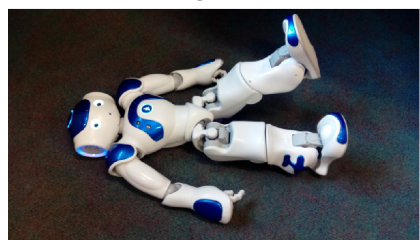

(b) Hip Abduction Laying

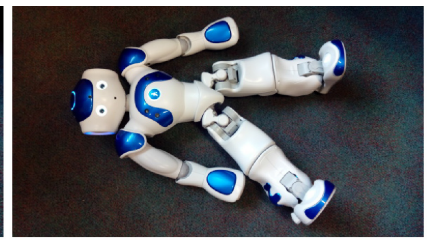

(e) Hip Extension Easy

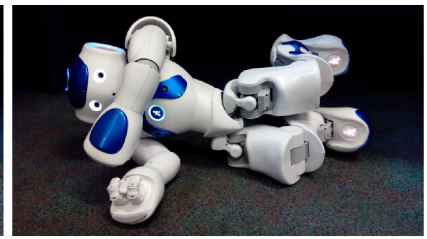

(h) Hip Knee Flexion Lifting

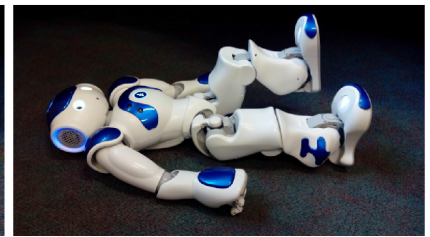

(k) Quads over Roll

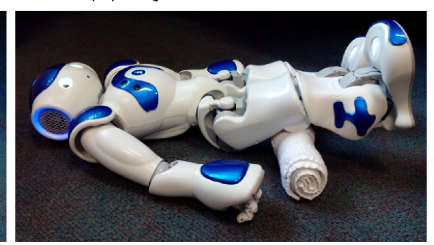

(c) Hip Abduction on Side

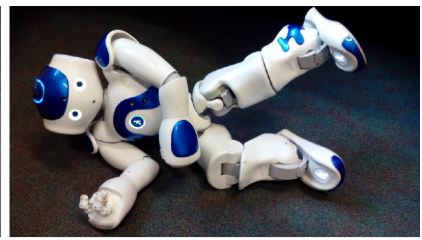

(f) Hip Extension Hard

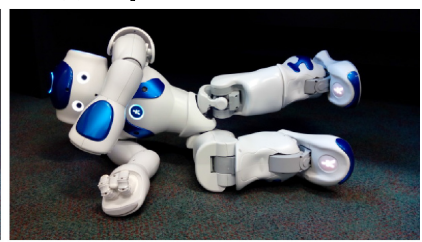

(i) Knee Extension on Side

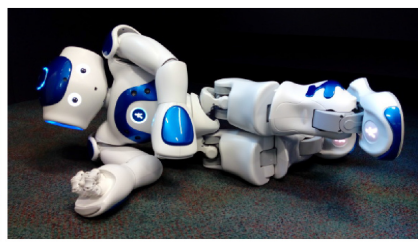

(l) Static Quads

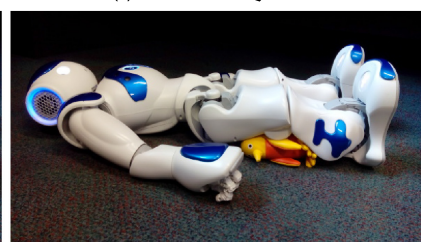

Fig. 5. Rehabilitation exercises executable from a lying-down position. (a) Bridge: Strengthening exercise for the hip extension muscles. (b) Hip Abduction Lying: Strengthening exercise for hip abduction muscles. (c) Hip Abduction on Side: Progression of hip abduction lying. (d) Single Bridge: Progression of double leg bridge. (e) Hip Extension Easy: Strengthening exercise for the hip extension muscles. This is easier than bridges and can be done with children who are not allowed to take weight through the legs. (f) Hip Extension Hard: Progression of Hip Extension Easy. Keeping the knee straight while extending the hip makes this exercise harder. (g) Hip Knee Flexion Sliding: Strengthening exercise for the hip flexors that can also be used to encourage increased range of movement at the hip and knee. The weight of the leg is supported by the bed. (h) Hip Knee Flexion Lifting: Strengthening exercise for the hip flexors and improving range of movement at the hip and knee. (i) Knee Extension on Side: In this exercise, gravity is eliminated, meaning it is an easier exercise for strengthening the muscles that extend the knee. (j) Leg Raises: Strengthening exercise for hip flexors and quadriceps. (k) Quads over Roll: Strengthening exercise for the hip extensor muscles. (I) Static Quads: This exercise is used to start practising engaging the muscles that extend the knee. It is easier than Quads over Roll.

a text-based interface, avoiding any code modifications between sessions. This implementation allows a session to be configured by selecting and sequencing exercises in the system, together with the number of sets, repetitions, and execution speed. Other parameters entered to personalise the session are the patient's and caregiver's names. Configuration of the SAR is currently done via 
a text file edited by a technology developer on behalf of the therapist. Development of a caregiver's interface is currently underway and will soon be deployed as part of the system.

\subsection{Rehabilitation Exercises}

All rehabilitation exercises and activities described in Section 4.2 are standard exercises in existing rehabilitation programs (integration requirement). However, changes to the initial design of some exercises were required to accommodate stability, robustness and endurance requirements. For example, the sit-to-stand exercise was originally designed to work with a seat, requiring prepositioning before exercise execution. However, due to an observed high risk of failure in Phase 1 (e.g., movement of the seat or incorrect positioning), the activity was redesigned in consultation with therapists to incorporate a crouching action instead. This was more reliable and simpler to initiate.

Walking exercise demonstrations were trialled in Phase 1 but not included in the Phase 2 prototype. In line with Malik et al. [20], therapists deemed the crouching gait of the NAO robot as not appropriate for demonstration to patients. Furthermore, Phase 1 highlighted issues with both the speed and stability of NAO's walk. For example, the toy-relay activity scenario was designed to motivate walking in the patient by having the robot issue instructions and through face tracking and motivational utterances provide patients a sense of being monitored and encouraged during the activity (Figure 4).

\subsection{Activity Execution Order}

It was observed during rehabilitation sessions in Phase 1 that therapists often wanted to modify the schedule of exercises to better adapt to the patient's mood and energy levels. This was easily facilitated in Phase 1 with technology developers in place but required careful consideration for Phase 2's stand-alone system. Providing therapists the ability to schedule the execution order of rehabilitation activities was thus deemed central to the flexibility requirement but needed careful balancing with stability and endurance requirements of the system. For example, while some therapists expressed a desire for on-line reordering of activities during sessions, this was not incorporated into our initial Phase 2 prototype owing to increased risk of failure during transitions between some exercise poses. This decision was supported by observations of care delivery in Phase 1, which revealed a general tendency for therapists to maintain the basic order of exercises and, in particular, to group exercises based on the required posture or stage of the session (e.g., lying down vs. standing up, muscle strengthening vs. relaxing).

\subsection{Exercise Speed}

The speed of exercise execution was noted as something that needed to be changeable during sessions. Phase 1 made clear that not all patients perform exercises at the same speed and during intensive rehabilitation are likely to progress to more capable levels. Physiotherapists request that children perform exercises at different speeds based on their clinical observations of exercise performance. This may include performing some exercises faster or slower, or holding a position longer. Therefore, all the exercises have been programmed for three different speeds, allowing therapists the ability to select a speed during preconfiguration and during the execution of an exercise set to support the adaptability requirement (for more details, see Section 5.7).

Static Quads is the fastest exercise in which each repetition in the fast-speed setting takes 2 seconds, dropping to 5 seconds in the slow-speed setting. Hip Abduction is the slowest exercise, in which each repetition takes 7 seconds on the fast-speed setting, increasing to 15 seconds when set to slow speed. Exercise speeds were validated based on initial observations of the robot performing the exercises and then clinical observation of a child performing exercises with the robot. 
Physiotherapists provided feedback to technology developers to make speed adjustments based on this.

\subsection{Human-Robot Interaction}

5.5.1 Robot Gestures and Speech. Observations during Phase 1 and early testing of the Phase 2 prototype highlighted a need for speech at frequent and intermittent points to avoid long periods of silence. In Phase 1, this was easily accounted for through Wizard-of-Oz operations, but the stand-alone requirement forced the Phase 2 prototype to be equipped with an extensive scripted list of utterances, selected randomly, for specific activity scenarios. Therapists suggested the inclusion of motivational statements and reminders of important aspects of the movement to maximise therapeutic benefit. Motivational statements such as "Keep it Going!" or "Every exercise we do gets us closer to my awesome dance moves!" are randomly selected and interleaved with exercise-specific reminders such as "Can you lift your bottom any higher?" or "Keep your toes pointing up!". Constant feedback is also provided during exercise execution by counting each repetition aloud.

Due to robustness and reliability considerations in the Phase 2 prototype, no patient progress monitoring has been incorporated into SAR feedback to patients. Thus, statements are designed to be relevant to the specific exercise but not specific to the particular patient's current actions or progress. While therapist feedback made clear a desire for patient monitoring to inform the delivery of statements, this was not regarded as a prerequisite to clinical deployment.

Along with speech, animated gestures and actions have been incorporated into the SAR. Chidambaram et al. [6] studied how appropriately designed vocal and nonverbal cues can increase compliance in people when instructed by a robot. Accordingly, we have incorporated built-in gestures for animated speech to enhance compliance and the overall authenticity of interactions with patients.

5.5.2 Speech Recognition. The challenges of speech recognition with social robots such as NAO, and for voice recognition with children more generally, are well documented in the HRI literature [14]. Pelikan and Broth [28], for example, note issues associated with the required turn taking between robot and human when delivering speech, which users often find difficult to adapt to. Insufficient loudness of voiced responses or unexpected statements provided by human users pose significant challenges for SARs seeking to foster natural and authentic interactions with users.

Phase 1 confirmed all of these issues as significant challenges but also highlighted issues more specific to the clinical context. For example, errors in speech recognition would cause NAO to provide inappropriate responses owing to misclassification of responses to questions such as "How are you doing?."Negative patient responses were sometimes classified as positive (and vice versa), potentially impeding the SAR's primary role as a motivator and companion. This was exacerbated by the relatively young age of children and, in some cases, speech impediments relating to their disability. A lack of response to a patient's answer would also result in long periods of silence, often requiring a supervising adult to intervene and repeat the command.

Such challenges, however, were countered by Phase 1 observations that children reacted positively when the robot did respond appropriately. The incorporation of limited speech recognition was thus deemed important to realise interaction and responsiveness requirements. To preserve stand-alone and integration requirements of the system, bidirectional communication was governed by specific structural choices to constrain possible responses and to ensure robustness to misclassified utterances. These choices included the following:

- Prompting users only for simple, specific one-word verbal responses such as: "When you're ready to start, just say 'go!"' and/or asking scripted questions with a constrained set of possible one-word responses (e.g., Yes/No). 
(a)

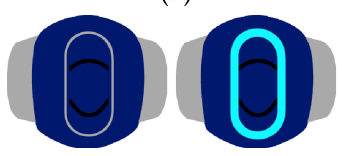

(b)

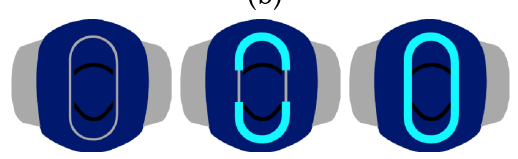

(c)

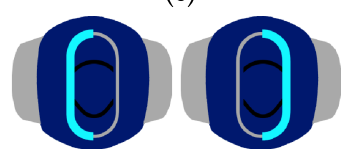

Fig. 6. LED effects (grey and thin line when LEDs are off, cyan or thick line when LEDs are on). (a) Prompting a patient/caregiver head-tap. (b) Indicating system is paused. (c) Indicating a system setup in progress.

- Providing nonverbal tactile-based interaction alternatives. For example: "Sorry, I didn't hear you! You can also tap my head to continue."

- Providing speech recognition with an array of possible responses from which to base speech classification. For example: "Yes," "Yeah," "Sure," "Okay," "Yep."

- Capping the waiting period for a patient response at 2 seconds to ensure that no undue pressure was placed on the patient to provide a response. A lack of response would simply be followed by a generally relevant statement before continuing execution of the scenario. A 2-second listening time was chosen from empirical observations in Phase 1.

A limited number of more open interactions were also included to allow patients the opportunity to engage more freely and express feeling and emotion (e.g., "How are you doing?"). Such interactions were included, in part, to allow supervising caregivers (and researchers) a chance to gauge the patient's emotional state during the session. SAR responses to patient answers were designed to be generally relevant rather than response specific. For example, a patient's response, either negative or positive, might be followed by the generic statement: "I am having a great time doing these exercises with you."

\subsection{Visual Cues}

To support interaction and stand-alone requirements, NAO provides multiple LED outputs to prompt user input and convey the system state. LEDs around the three head buttons of the NAO are used extensively to cue required button presses to confirm progression to the next activity. LEDs blink at $2 \mathrm{~Hz}$, cueing the need for the head to be tapped either between exercise sets or when changing activity scenarios. Phase 1 indicated that visual cueing greatly improved the ability and confidence of people to perform the task. Full blinking of head LEDs is used to cue confirmation of progression to the next activity (Figure 6(a)). Other patterns of LED flashing convey that the system is setting up (Figure 6(c)) or is in a paused state (Figure 6(b)).

Additional LED cueing on either side of NAO's head conveys the expectation of a verbal input, most commonly as an alternative to head tapping for confirming progression to the next activity.

\subsection{Tactile Interface}

Use of the NAO's head-based tactile sensors provides caregivers and patients an alternative to speech for SAR interaction. In therapy sessions, patients can use the tactile interface when prompted to continue to the next activity or to start another set of repetitions. To ensure simplicity for patients, this is achieved via a single tap of any of the three buttons (Figure 7(a)).

To support online adaptability and configurability requirements, head taps were also used to provide caregivers the ability to adjust activity settings. Most prevalent in Phase 1 observations were scenarios in which patient performance required adjustment of exercise speed or pausing of the session to accommodate unpredictable actions. 

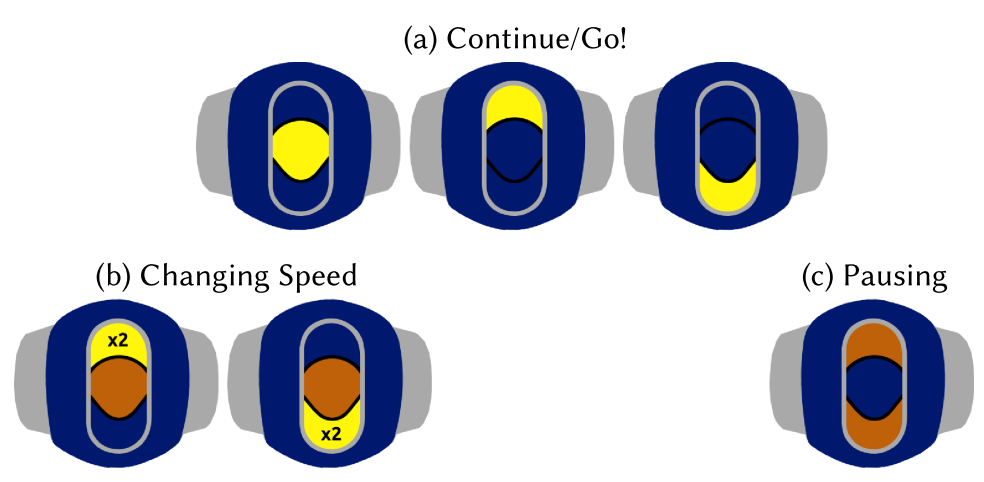

Fig. 7. Tactile interface. (a) Continue/Go!: A single tap on any of the three tactile buttons (middle, front, or rear) when requested by the SAR to continue. (b) Changing Speed: One finger middle button long press while second finger double tapping the front bottom to go faster or double tapping the rear button to go slower. (c) Pausing the Robot: Long press to front and rear buttons at the same time.

Speed adjustments are achieved using a sustained press of the NAO's middle head touch sensor followed by a double tap of the front sensor to slow down the exercise or to the rear sensor to speed it up (Figure 7(b)). To pause the robot, the rear and the front button are long pressed at the same time (Figure 7(c)). Robot adjustments are less simple than head taps to prevent readjustments by mistake (robustness requirement).

\subsection{Human-Assisted Activities}

While NAO offers a high degree of autonomy, Phase 1 observations highlighted limitations in the context of its ongoing therapeutic use. Physical constraints and other system uncertainties limit the ability of the robot to perform certain exercises, attain certain postures, or position itself with respect to supportive auxiliary aids. Even where autonomy may be possible, motor wear-and-tear, uncertainty of success, and time costs associated with completing some actions autonomously motivated the use of human assistance in certain instances to meet robustness and stability requirements.

The inclusion of robot capabilities needing human assistance, while unavoidable, required careful consideration. To meet integration and stand-alone operation requirements, the inclusion of activity scenarios requiring caregiver assistance needed to be complementary to existing caregiver tasks-in particular, preserving the caregiver's focus on the needs of the patients. In consultation with therapists, the following human-assisted capabilities have been implemented in the Phase 2 prototype:

Positioning: Activity scenarios can be done in a range of different places and positions: On the floor, on a table, lying down, standing up, etc. While NAO can stand up or lie down by itself, manual repositioning, in which the therapist lifts and places the robot close to the patient, is quicker, less error prone, and better reduces wear-and-tear (Figure 8(a)) than having the robot position itself.

Placing auxiliary aid: Quads over Roll and Static Quads are the two exercises in which, as with the patient, a small rolled towel is placed under the leg of the robot (Figure 8(b)). The robot will ask explicitly for this kind of assistance:

"For Quads over Roll, we will need to roll two towels. One big for you and a little one for me! We have to put the towel under our left knee." 
(a) Positioning the robot

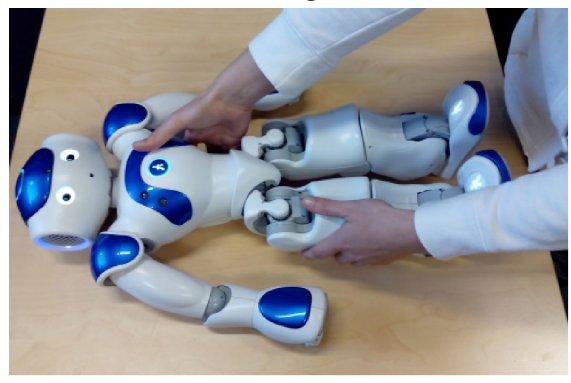

(c) Posture

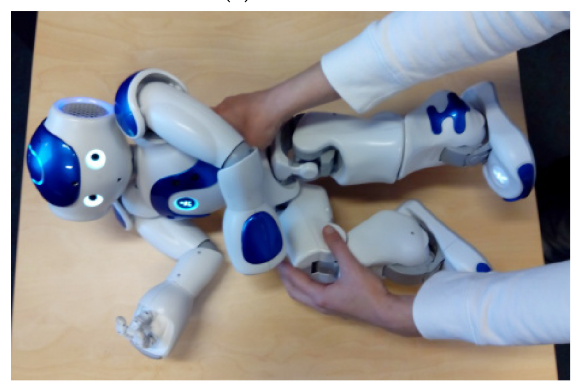

(b) Placing auxiliary aids

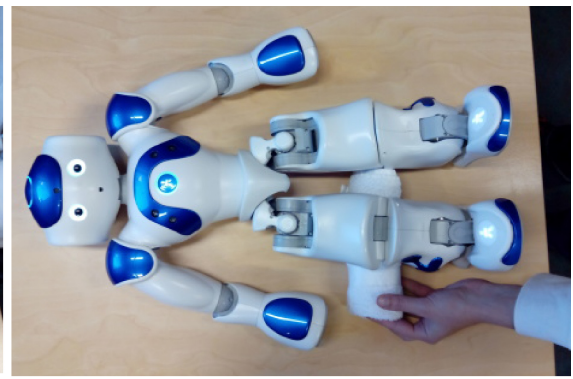

(d) Helping to keep pace

Fig. 8. Examples of human-assisted activities.

Posture: Hip Abduction on Side, Hip Extensions, and Knee Extension on Side are exercises for which the robot needs to be rolled onto its side (Figure 8(c)). As with auxiliary aids, the robot asks explicitly for this kind of assistance:

"For this exercise, I will need your help! I will need you to roll me onto my right side. Can you do that for me?"

Keeping pace: Between exercises, the SAR lets the patient rest. A head tap (Figure 8(d)) is used to indicate progression to the next activity. Head taps are also used to confirm progress during instructional activities, such as sit-to-stands or toy relay.

"Say Go! Or tap my head when you are ready to start the next set."

Our preliminary results showed that the amount of time physiotherapists had to deal with the robot did not negatively impact patient sessions [22]. Phase 2 evaluation is closely examining time costs and frequency of such requests with respect to the overall perceived benefits of the system. We discuss this further in Section 6.4.

\section{INITIAL CLINICAL DEPLOYMENT: SYSTEM AND IN SITU DESIGN CRITIQUE}

Initial Phase 2 testing has commenced in preparation for a planned clinical evaluation of the system. In this section, we present our methodology for evaluating the Phase 2 system as we develop the SAR for clinical trials. Here, we focus primarily on operational aspects of the SAR with respect to the requirements and design decisions outlined in previous sections. We also present initial user response data from therapists and parents who have observed the SAR in the clinical care of patients. Due to the early stage of clinical testing, we defer a comprehensive evaluation of the SAR's 


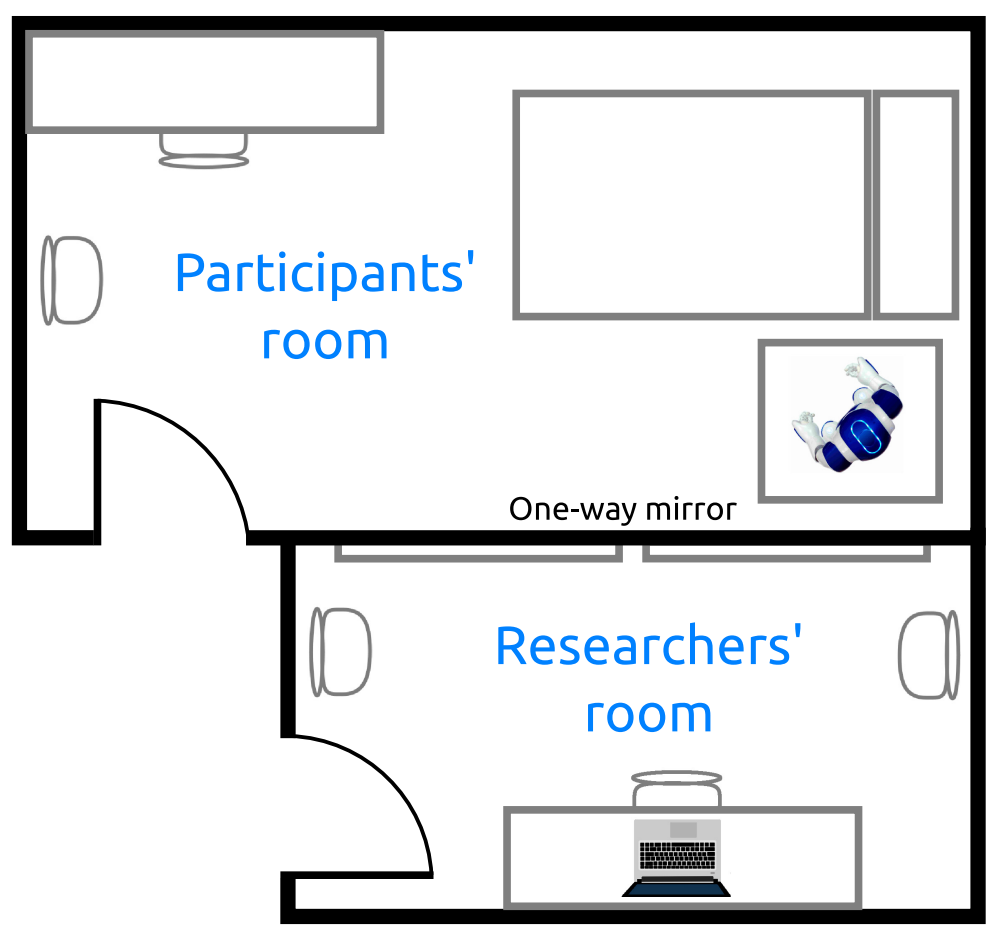

Fig. 9. Study setting floor plan.

perceived therapeutic benefits and patient/parent/therapist perceptions until the completion of Phase 2 testing.

\subsection{Phase 2 Testing Setup}

Phase 2 clinical sessions with the robot are conducted in a consultation room at the rehabilitation clinic of the Royal Children's Hospital, Melbourne, Australia. Observing investigators reside in an adjacent observation room with a one-way mirror (see Figure 9). The patient, a therapist and the SAR are in the participants' room. Parents can observe the session from either of the two rooms. All participants are informed that sessions are being observed by research team members. Preconfiguration of the system is performed by a research team member. Configuration options are communicated to the research team member by the treating therapist prior to each session.

Before starting the session, the robot is placed in a crouched position on a tabletop next to the bed and the attending therapist receives a 5-minute informal introduction to the system. In this introduction, it is explained that the robot will work autonomously and will be able to recover from some failures; however, it may ask for help for particular positioning requirements or request head taps to confirm session progression.

The session starts with the robot greeting the patient and introducing itself. NAO then commences the patient's preconfigured exercise program, as described in Section 4.2.

NAO's software currently runs off a laptop with a wireless connection to the robot. During each session, an attending research engineer monitors the software in the adjacent observation room and interacts with the system only if necessary (i.e., a system failure requiring a reset of the system). All operational requirements are thus handled by attending caregivers and the patient. 
Our protocol allows engineer intervention to occur only when a system error or issue is disrupting the session and is easily recoverable in situ. All such instances are logged.

\subsection{Data Collection}

During each session, observations on the system performance, usability, and interactions among participants and the robot are recorded by observing research team members. System logs for each session are also recorded, capturing exercise configuration, completed exercises (by the robot), user prompts, number of requests for help, and time required for needs to be met.

A key focus of Phase 2 testing is the evaluation of the SAR's perceived utility, ease of use, and participants' trust of the robot as a therapeutic device. To this end, survey response data is collected using adapted versions of the robot acceptance questionnaire originally proposed by Heerink et al. [11]. Responses are recorded using a Likert scale, with specific versions of the questionnaire used for each of the three participant groups (patients, parents, therapists).

Tables 2 and 3 present the adapted survey questions for physiotherapists and parents, respectively, along with initial responses (discussed further below). The questionnaire is divided into different categories: Anxiety (ANX1, ANX2), Attitude (ATT), Facilitating Conditions (FC), Intention to Use (ITU), Perceived Adaptability (PAD), Perceived Ease of Use (PEOU), Perceived Usefulness (PU), Trust (TR), and Social Influence (SI). Anxiety category is divided in two parts to better understand the extent to which participants were anxious about their safety with the robot as distinguished from anxieties associated with using the system correctly and without damaging it.

Participants are also asked open questions seeking feedback on strengths and weaknesses of the system, desirable features currently not present, and their impressions of trust and benefit. Owing to the young age and cognitive impediments of many of the patients expected to be recruited in Phase 2 testing, only limited survey feedback is expected from patients, as determined by physiotherapist clinical judgement.

\subsection{Preliminary Session Results}

6.3.1 System Performance. At the time of writing, our Phase 2 prototype has led 14 observed sessions of up to 30 minutes each. Table 1 provides a structured overview of the 14 sessions, indicating which exercises were performed, the duration of each session, exercises completed, and any system disruptions that may have occurred.

Of the 14 sessions conducted, 9 sessions finished with patients completing all prescribed exercises. Two of the five sessions not completed fully were shortened by the attending physiotherapist (Sessions 4 and 5) based on clinical judgement. In Session 4, the toy relay was excluded due to patient fatigue (though the patient remained positive throughout the session), and in Session 5, two programmed exercises were not conducted owing to the patient's perceived lack of stamina. One session was aborted owing to an unrecoverable system error (Session 2), causing the last prescribed exercise for the session to be completed without the robot.

Sessions 12 and 14 involved recoverable system disruptions. In Session 12, a loss of stability occurred during the final dance behaviour; in Session 14, a loss of power (after back-to-back sessions) required engineer intervention to resolve.

Two sessions were aborted owing to patients' explicit expression of dislike of the SAR. In Session 3, a 3-year-old patient expressed fear of the robot owing to its loudness, causing an immediate halt to the session. In the second case, Session 10, a teenage patient expressed a clear dislike of the robot, invoking a premature stop to the session. Therapist feedback noted that the patient has a history of noncompliance in therapy sessions. These events reflect a clear diversity of patient needs and are informative to future development and testing of the system. 
Table 1. Rehabilitation Sessions in Phase 2 Summarised

\begin{tabular}{|c|c|c|c|c|c|c|}
\hline No & Patient & Exercises programmed & Exercises completed & Duration & System Disruptions & Comments \\
\hline 1 & P-1 & $\begin{array}{l}\text { Quads over Roll } \\
\text { Bridge } \\
\text { Hip Abductions Lying }\end{array}$ & $\begin{array}{l}\text { Quads over Roll } \\
\text { Bridge } \\
\text { Hip Abductions Lying }\end{array}$ & $\mathrm{N} / \mathrm{A}$ & & $\begin{array}{l}\text { Patient expressed positive } \\
\text { attitudes and showed focus } \\
\text { on the SAR. }\end{array}$ \\
\hline 2 & P-2 & $\begin{array}{l}\text { Quads over Roll } \\
\text { Bridge } \\
\text { Hip Knee Extension }\end{array}$ & $\begin{array}{l}\text { Quads over Roll } \\
\text { Bridge }\end{array}$ & $\mathrm{N} / \mathrm{A}$ & $\begin{array}{l}\text { The last exercise was not } \\
\text { executed owing to an error } \\
\text { in the system. }\end{array}$ & $\begin{array}{l}\text { The patient did not like } \\
\text { the robot. }\end{array}$ \\
\hline 3 & P-3 & $\begin{array}{l}\text { Hip Knee Flexion Lifting } \\
\text { Toy Relay }\end{array}$ & & $\mathrm{N} / \mathrm{A}$ & & $\begin{array}{l}\text { Session aborted. Robot was } \\
\text { too loud, upset the patient. }\end{array}$ \\
\hline 4 & $\mathrm{P}-4$ & $\begin{array}{l}\text { Static Quads } \\
\text { Quads over Roll } \\
\text { Single Bridge } \\
\text { Hip Knee Flexion Sliding } \\
\text { Toy Relay }\end{array}$ & $\begin{array}{l}\text { Static Quads } \\
\text { Quads over Roll } \\
\text { Single Bridge } \\
\text { Hip Knee Flexion Sliding }\end{array}$ & $19: 41$ & & $\begin{array}{l}\text { Patient proactively helped } \\
\text { the robot when required. } \\
\text { Due to patient's fatigue, } \\
\text { physiotherapist shortened } \\
\text { the session. Patient happy to } \\
\text { do another session. }\end{array}$ \\
\hline 5 & P-5 & $\begin{array}{l}\text { Quads over Roll } \\
\text { Bridge } \\
\text { Hip Abduction on Side } \\
\text { Leg Raises }\end{array}$ & $\begin{array}{l}\text { Quads over Roll } \\
\text { Bridge }\end{array}$ & $10: 50$ & & $\begin{array}{l}\text { Patient showed enthusiasm } \\
\text { for a session with the SAR. } \\
\text { Patient's frustration } \\
\text { with the exercises shortened } \\
\text { the session. }\end{array}$ \\
\hline 6 & $\mathrm{P}-4$ & Toy Relay & Toy Relay & $23: 23$ & & \\
\hline 7 & P-6 & $\begin{array}{l}\text { Static Quads } \\
\text { Hip Abductions Lying } \\
\text { Toy Relay }\end{array}$ & $\begin{array}{l}\text { Static Quads } \\
\text { Hip Abductions Lying } \\
\text { Toy Relay }\end{array}$ & $16: 19$ & & $\begin{array}{l}\text { Patient expressed positive } \\
\text { attitudes towards the robot, } \\
\text { enjoyment and excitement. }\end{array}$ \\
\hline 8 & P-6 & $\begin{array}{l}\text { Static Quads } \\
\text { Quads over Roll } \\
\text { Leg Raises } \\
\text { Toy Relay }\end{array}$ & $\begin{array}{l}\text { Static Quads } \\
\text { Quads over Roll } \\
\text { Leg Raises } \\
\text { Toy Relay }\end{array}$ & $24: 52$ & & $\begin{array}{l}\text { Patient showed focus on the } \\
\text { robot. Patient happy to } \\
\text { do another session. }\end{array}$ \\
\hline 9 & P-6 & $\begin{array}{l}\text { Static Quads } \\
\text { Quads over Roll } \\
\text { Leg Raises } \\
\text { Toy Relay }\end{array}$ & $\begin{array}{l}\text { Static Quads } \\
\text { Quads over Roll } \\
\text { Leg Raises } \\
\text { Toy Relay }\end{array}$ & $25: 42$ & & $\begin{array}{l}\text { Patient expressed positive } \\
\text { attitudes towards the robot, } \\
\text { smiled and interacted with } \\
\text { robot. }\end{array}$ \\
\hline 10 & P-7 & $\begin{array}{l}\text { Sit-to-Stands } \\
\text { Toy Relay }\end{array}$ & & $\mathrm{N} / \mathrm{A}$ & & $\begin{array}{l}\text { Session aborted. Patient } \\
\text { noncompliant in therapy } \\
\text { sessions. }\end{array}$ \\
\hline 11 & P-8 & $\begin{array}{l}\text { Static Quads } \\
\text { Quads over Roll } \\
\text { Leg Raises }\end{array}$ & $\begin{array}{l}\text { Static Quads } \\
\text { Quads over Roll } \\
\text { Leg Raises }\end{array}$ & $17: 15$ & & $\begin{array}{l}\text { Patient showed enjoyment } \\
\text { and proactively helped the } \\
\text { robot when required. }\end{array}$ \\
\hline 12 & P-8 & $\begin{array}{l}\text { Static Quads } \\
\text { Quads over Roll } \\
\text { Leg Raises }\end{array}$ & $\begin{array}{l}\text { Static Quads } \\
\text { Quads over Roll } \\
\text { Leg Raises }\end{array}$ & $16: 55$ & $\begin{array}{l}\text { Robot fall during the final } \\
\text { dance routine. No technical } \\
\text { intervention was required. }\end{array}$ & $\begin{array}{l}\text { Patient showed focus on the } \\
\text { robot. Patient happy to } \\
\text { do another session. }\end{array}$ \\
\hline 13 & $\mathrm{P}-8$ & $\begin{array}{l}\text { Static Quads } \\
\text { Quads over Roll } \\
\text { Leg Raises }\end{array}$ & $\begin{array}{l}\text { Static Quads } \\
\text { Quads over Roll } \\
\text { Leg Raises }\end{array}$ & $16: 50$ & & $\begin{array}{l}\text { Patient showed focus on the } \\
\text { robot and expressed positive } \\
\text { attitudes when interacting. }\end{array}$ \\
\hline 14 & P-9 & $\begin{array}{l}\text { Static Quads } \\
\text { Quads over Roll } \\
\text { Bridge } \\
\text { Hip Abductions Lying } \\
\text { Hip Knee Flexion Sliding } \\
\text { Sit-to-Stands }\end{array}$ & $\begin{array}{l}\text { Static Quads } \\
\text { Quads over Roll } \\
\text { Bridge } \\
\text { Hip Abductions Lying } \\
\text { Hip Knee Flexion Sliding } \\
\text { Sit-to-Stands }\end{array}$ & $31: 35$ & $\begin{array}{l}\text { Battery drainage. } \\
\text { Engineer intervention was } \\
\text { required to restart the } \\
\text { system. }\end{array}$ & $\begin{array}{l}\text { Teenager patient did the } \\
\text { session by her own, } \\
\text { physiotherapist helped at } \\
\text { the beginning. }\end{array}$ \\
\hline
\end{tabular}

Note: Fourteen sessions, with 9 different patients. The exercises programmed are chosen by the patient's physiotherapist. Duration of the rehabilitation session, including introductory speech and farewell dance, in mm:ss format. 
Table 2. Acceptance Questionnaire for Physiotherapists with Their Initial Responses

\begin{tabular}{|c|c|c|c|c|c|c|}
\hline \multirow[b]{2}{*}{ Construct } & \multirow[b]{2}{*}{ No } & \multirow[b]{2}{*}{ Question } & \multicolumn{4}{|c|}{ Responses } \\
\hline & & & PT-1 & PT-2 & PT-3 & PT-4 \\
\hline \multirow[t]{2}{*}{ ANX1 } & 1 & I would be afraid to make mistakes using the robot. & 1 & 2 & 3 & 3 \\
\hline & 2 & I would be afraid to break something when using the robot. & 3 & 2 & 4 & 5 \\
\hline \multirow[t]{2}{*}{ ANX2 } & 3 & I find the robot scary. & 1 & 2 & 1 & 1 \\
\hline & 4 & I find the robot intimidating. & 1 & 2 & 2 & 1 \\
\hline \multirow[t]{2}{*}{ ATT } & 5 & I think it's a good idea to use the robot. & 5 & 4 & 4 & 4 \\
\hline & 6 & The robot would make therapy sessions more interesting. & 5 & 4 & 4 & 4 \\
\hline \multirow[t]{2}{*}{ FC } & 7 & I have everything I need to make good use of the robot. & 4 & 3 & 3 & 4 \\
\hline & 8 & I know enough of the robot to make good use of it. & 4 & 3 & 2 & 3 \\
\hline \multirow[t]{3}{*}{ ITU } & 9 & $\begin{array}{l}\text { If I have access to the robot, I think I'll use it during the next } \\
\text { therapy sessions. }\end{array}$ & 4 & 4 & 3 & 4 \\
\hline & 10 & $\begin{array}{l}\text { If I have access to the robot, I am certain to use it in the next } \\
\text { therapy sessions. }\end{array}$ & 4 & 3 & 3 & 3 \\
\hline & 11 & $\begin{array}{l}\text { If I have access to the robot, I'm planning to use it during the } \\
\text { next therapy sessions. }\end{array}$ & 4 & 4 & 3 & 3 \\
\hline \multirow[t]{3}{*}{$\mathrm{PAD}$} & 12 & I think the robot can be adaptive to what I need. & 3 & 4 & 2 & 2 \\
\hline & 13 & $\begin{array}{l}\text { I think the robot will only do what I need at that particular } \\
\text { moment. }\end{array}$ & 3 & 3 & 2 & 4 \\
\hline & 14 & I think the robot will help me when I consider it to be necessary. & & 4 & 3 & 4 \\
\hline \multirow[t]{5}{*}{ PEOU } & 15 & I think I will know quickly how to use the robot. & 5 & 4 & 3 & 5 \\
\hline & 16 & I find the robot easy to use. & 5 & 4 & 3 & 4 \\
\hline & 17 & $\begin{array}{l}\text { I think I will be able to use the robot without any help if I have } \\
\text { been trained. }\end{array}$ & 5 & 4 & 3 & 4 \\
\hline & 18 & $\begin{array}{l}\text { I think I will be able to use the robot when there is someone } \\
\text { around to help me. }\end{array}$ & 5 & 4 & 4 & 5 \\
\hline & 19 & $\begin{array}{l}\text { I think I will be able to use the robot when I have a good } \\
\text { manual. }\end{array}$ & 5 & 4 & 3 & 5 \\
\hline \multirow[t]{3}{*}{ PU } & 20 & I think the robot is useful to help in paediatric therapy. & 5 & 4 & 4 & 4 \\
\hline & 21 & $\begin{array}{l}\text { It would be convenient to have the robot for therapy sessions } \\
\text { with children. }\end{array}$ & 5 & 4 & 4 & 4 \\
\hline & 22 & $\begin{array}{l}\text { I think the robot can help me with many things during } \\
\text { paediatric sessions. }\end{array}$ & 4 & 4 & 3 & 4 \\
\hline \multirow[t]{4}{*}{ SI } & 23 & I think the staff would like me using the robot. & 3 & 3 & 4 & 4 \\
\hline & 24 & I think parents would like me using the robot. & 5 & 4 & 4 & 3 \\
\hline & 25 & I think patients would like me using the robot. & 5 & 4 & 3 & 4 \\
\hline & 26 & I think it would give a good impression if I should use the robot. & 4 & 3 & 3 & 4 \\
\hline \multirow[t]{2}{*}{ TR } & 27 & I would trust the robot if it gave me advice. & 3 & 3 & 2 & 2 \\
\hline & 28 & I would follow the advice the robot gives me. & 3 & 4 & 3 & 2 \\
\hline
\end{tabular}

Note: The questionnaire is divided into different constructs: Anxiety (ANX1, ANX2), Attitude (ATT), Facilitating Conditions (FC), Intention to Use (ITU), Perceived Adaptability (PAD), Perceived Ease of Use (PEOU), Perceived Usefulness (PU), Trust (TR), and Social Influence (SI). Likert Scale: $1=$ Strongly Disagree; $2=$ Disagree; $3=$ Neutral; $4=$ Agree; $5=$ Strongly Agree.

6.3.2 Therapist/Parent Feedback. In early Phase 2 testing, survey responses from 4 different physiotherapists have been recorded upon completion of their first session interacting with the SAR. As participant numbers are small, we present the raw quantitative data provided in Table 2 and an overview of the open question responses.

Inspection of these early survey responses show that 3 of the 4 recruited physiotherapists perceived the system as easy to use. However, the fourth physiotherapist (PT-3) expressed mostly 
neutral opinions of the system's usability and disagreement about having enough knowledge of the robot to make use of it effectively. Notably, physiotherapists had only a brief introduction to the SAR at the beginning of their first session with it; however, they were observed to exhibit competence interacting with and operating the SAR.

All physiotherapists expressed positive attitudes towards using the robot in rehabilitation therapy (ATT). In response to questions regarding the SAR's perceived usefulness (PU), all therapists expressed either agreement or strong agreement that the robot is convenient and useful for paediatric rehabilitation (PU).

Responses to questions of intention to use (ITU) the SAR in future sessions presents a less clear picture from early data collection. While 3 out of 4 therapists agree that they would consider using the SAR during the next therapy session, two of these therapists respond only neutrally to being certain of this. While no specific feedback elaborating on these responses was obtained, it is likely that confounding factors such as the unknown rehabilitation needs of future patients quite reasonably attenuated their certainty.

Physiotherapist responses to statements of trusting (TR) the robot's advice were, with the exception of one response (agree), either neural or in disagreement. No specific feedback was obtained to better understand these responses. We discuss this further below.

When asked about the most useful features of the robot, physiotherapists reported the SAR's ability to demonstrate exercises to patients and its motivational role in keeping the patient focused on each exercise to be most useful. However, therapists also noted deficits in the system's performance, including the SAR's lack of responsiveness to patient mood and performance and battery life in the context of back-to-back sessions (Session 14). Physiotherapists' reactions to the robot's lack of responsiveness suggests that they had expectations that the SAR would respond to the patient's mood. However, no physiotherapist explicitly expressed the desire for a feature to manually change the robot's behaviour to match the patient's current state.

As per our in situ design process, feedback from parents/guardians was also sought as part of preliminary Phase 2 testing. Raw survey responses for parents $(\mathrm{N}=4)$ attending therapy sessions are presented in Table 3. Notably, all parents expressed overwhelming agreement to statements reflecting the SAR's PU. All strongly agreed that the SAR is useful in their child's therapy and all agreed that the robot can help their child with many things. Parents also reported ATTs to using the SAR in their child's rehabilitation therapy. Of particular interest for future testing of the SAR is parents' perceptions of the SAR's usability (PEOU). Three parents responded positively to the robot being easy to use and to feeling confident in using the system themselves. One parent (G-4) expressed mostly neutral responses to PEOU questions, although also strongly disagreed to being able to use the SAR without any help. Notably, all except one parent also expressed disagreement or neutrality about having enough knowledge to make good use of the SAR. As future testing of the SAR intends to allow parents to operate the SAR without therapist supervision, these results are both encouraging and informative, indicating that with more targeted training and familiarisation, it is reasonable to expect parents to feel capable and comfortable operating the SAR on their own. In contrast with the physiotherapist's responses, parents in general strongly agree that they would trust (TR) and follow the robot's advice.

In open feedback, 3 out of the 4 parents specifically noted the the robot helped keep their child focused on completing the exercises. These statements included the following: "[Daughter] seemed to respond really well and her mind was taken off [the rehabilitation session] with the robot"; and "The robot was useful because it had my child's attention the whole time."Observational data corroborated these perceptions, with patients exhibiting high focus on the SAR during the rehabilitation session. Notably, two parents of female patients noted that they would prefer gender-neutral colouring for NAO. 
Table 3. Acceptance Questionnaire for Guardians with Initial Responses

\begin{tabular}{|c|c|c|c|c|c|c|}
\hline \multirow[b]{2}{*}{ Construct } & \multirow[b]{2}{*}{ No } & \multirow[b]{2}{*}{ Question } & \multicolumn{4}{|c|}{ Responses } \\
\hline & & & G-1 & G-2 & G-3 & G-4 \\
\hline \multirow[t]{2}{*}{ ANX1 } & 1 & I would be afraid to make mistakes using the robot & 2 & 5 & 1 & 3 \\
\hline & 2 & $\begin{array}{l}\text { I would be afraid to break something when using the } \\
\text { robot. }\end{array}$ & 1 & 3 & 5 & 4 \\
\hline \multirow[t]{2}{*}{ ANX2 } & 3 & I find the robot scary. & 1 & 5 & 1 & 1 \\
\hline & 4 & I find the robot intimidating. & 1 & 1 & 1 & 1 \\
\hline \multirow[t]{2}{*}{ FC } & 5 & I have everything I need to make good use of the robot. & 3 & 5 & 2 & 3 \\
\hline & 6 & I know enough of the robot to make good use of it. & 3 & 4 & 1 & 2 \\
\hline \multirow[t]{2}{*}{ ATT } & 7 & I think it's a good idea to use the robot. & 5 & 5 & 5 & 5 \\
\hline & 8 & $\begin{array}{l}\text { The robot would make my child's rehab sessions more } \\
\text { interesting. }\end{array}$ & 5 & 5 & 5 & 4 \\
\hline \multirow[t]{5}{*}{ PEOU } & 9 & I think I will know quickly how to use the robot. & 5 & 5 & 5 & 3 \\
\hline & 10 & I find the robot easy to use. & 5 & 5 & 5 & 3 \\
\hline & 11 & I think I can use the robot without any help. & 5 & 4 & 5 & 1 \\
\hline & 12 & $\begin{array}{l}\text { I think I can use the robot when there is someone around } \\
\text { to help me. }\end{array}$ & 5 & 5 & 5 & 3 \\
\hline & 13 & I think I can use the robot when I have a good manual. & 5 & 5 & 5 & 4 \\
\hline \multirow[t]{4}{*}{ PU } & 14 & I think the robot is useful for paediatric rehabilitation. & 5 & 5 & 5 & 5 \\
\hline & 15 & $\begin{array}{l}\text { It would be convenient to have the robot for therapy ses- } \\
\text { sions together with the physiotherapist. }\end{array}$ & 3 & 5 & 5 & 4 \\
\hline & 16 & $\begin{array}{l}\text { It would be convenient to have the robot for therapy ses- } \\
\text { sions when the physiotherapist is not in the session. }\end{array}$ & 4 & 5 & 5 & 3 \\
\hline & 17 & I think the robot can help my child with many things. & 5 & 5 & 5 & 4 \\
\hline \multirow[t]{2}{*}{ TR } & 18 & I would trust the robot if it gave me advice. & 4 & 5 & 5 & 5 \\
\hline & 19 & I would follow the advice the robot gives me. & 5 & 5 & 5 & 4 \\
\hline
\end{tabular}

Note: The questionnaire is divided into Different Constructs: Anxiety (ANX1, ANX2), Facilitating Conditions (FC), Attitude (ATT), Perceived Ease of Use (PEOU), Perceived Usefulness (PU), Trust (TR). Likert Scale: 1 = Strongly Disagree; $2=$ Disagree; 3 = Neutral; 4 = Agree; 5 = Strongly Agree.

6.3.3 Summary. Preliminary in situ testing indicates that the system is performing strongly on key metrics of acceptance in clinical practise: in particular, PU and PEOU. While session observations have highlighted areas of improvement for the system, discussed in the next section, therapists and parents respond almost universally positively to statements reflecting the SAR's usefulness and usability.

Preliminary results on trust provide less clarity. The issue of trust in HRI research is known to be complex and often difficult to interpret. Our survey responses reflect a level of distrust from therapists with respect to taking advice from the SAR. The short exposure time of the therapists to the SAR is a likely factor; however, it should also be noted that the survey questions refer only to the participant's own trust and willingness to follow the advice of the SAR separate from their trust in the system as a therapeutic aid. That therapists express more positivity towards statements reflecting their intention to use the SAR in future sessions provides some support for 
trust of the system in this respect. Notably, parents express a much higher degree of trust in the SAR's advice; however, it must be noted that this is likely to be conflated with trust that they may feel towards the therapist's clinical judgement to include the SAR in their child's therapy and the hospital's judgement in allowing the study to take place. It is thus difficult to draw any clear conclusions on trust from this data.

The robot has successfully led 9 of the 14 sessions through the same exercises that would usually be performed as per their rehabilitation program. Of the 5 noncompleted sessions, only one was due to an unrecoverable system error. While such incidents are undesirable, they are easily addressed and vindicate the in situ testing phase prior to clinical trials that we are undertaking.

Overall, preliminary observational and therapist feedback supports the assertion that the SAR is, more often than not, positively impacting the motivation of children to complete each exercise in full and correctly. However, more data is required to thoroughly evaluate this. Motivation to complete independent exercises is a known issue in physiotherapy practice (for both adults and children) and technological supports are showing promising results in improving compliance [18]. The particular value added by an embodied artificial agent such as NAO versus a virtual graphical body (for example, a video or animation) has also been previously explored, with evidence suggesting that participants perceive more social presence when interacting with a robot than with other virtual agents $[19,45]$, which, in turn, may lead to heightened motivation and emotional connection with the aid. Our own observations in both phases of design and development support this, with younger patients in particular exhibiting behaviours suggesting that they believe the robot is listening and responding to them.

\subsection{System Design Evaluation}

In this section, we discuss and critique specific design decisions (outlined in Section 5) based on the early Phase 2 testing outlined earlier. We discuss these in the context of developing the SAR for full clinical deployment.

6.4.1 Configurability. The SAR software was designed to support rapid configuration for new exercise sessions, allowing for the preselection and scheduling of exercises to perform, number of repetitions, speed of execution, entertainment modules, and patient and physiotherapist names. Configuration time was observed to take no more than 5 minutes; however, the current interface is text file based and thus not directly usable by therapists. While therapists were able to effectively communicate the session schedule to engineers via a text-based template, this was an inefficient process and will not scale to the ongoing clinical deployment of multiple robots or multiple patients with the same robot. To this end, a tablet-based interface for therapists and caregivers is under development allowing session histories to be stored and, importantly, the removal of research team members from the configuration process.

6.4.2 Stability and Robustness Versus Flexibility. The decision to fix the activity execution order during sessions was chosen to maintain stability and robustness requirements of the SAR by minimising posture and position changes. The low number of recorded system failures in Phase 2 testing supports this decision, with system failures to date occurring only during a dance (entertainment) scenario, owing to a system error that has been fixed and owing to power loss (see Table 1). However, flexibility is compromised and the inability to dynamically change exercise execution order was raised as a deficit of the current system design by therapists. One therapist suggested that the robot could ask the patient which exercise to do next, instead of following a prescribed order. Such flexibility is being considered within particular exercise subsets. For example, the system may allow therapists (or patients) to change execution order within a specific block of lower-body exercises. 
The SAR provides therapists the ability to dynamically alter the robot's exercise execution speed via a simple tactile interface; however, no recorded instances of its use were observed over the 14 Phase 2 sessions. Therapists have raised no specific concerns with the tactile interface and were observed to use this interface for other tasks, such as confirming progression to the next activity. Future work will focus more specifically on understanding the usability needs of this feature.

6.4.3 Speech and Interaction. No specific feedback about the animated speech was provided by participants; however, general observations of patient reactions suggested that the animated speech enhanced the SAR's authenticity with patients. The prototype has 20 preprogrammed phrases to encourage and motivate patients common for all exercises. Five specific instructional phrases are also programmed for each exercise and selected randomly. As noted previously, the system does not provide explicit monitoring; thus, any detailed feedback to patients regarding their performance is still assumed to be delivered by the physiotherapist or parent who is present. However, the feedback from NAO was sometimes reaffirmed by the parent or therapist to encourage the child. For example, one therapist said "See! NAO is also asking you to lift your bottom higher!"

Both developers and therapists noted a high degree of repetitiveness in the SAR's delivered statements, suggesting that the range of motivational phrases should be increased. However, this repetitiveness was not observed to impact negatively on engagement or compliance with the mostly young patients. It is interesting to note that therapists often deliver similarly frequent repeated statements as a means of reinforcing positive and important feedback. However, such phrases are typically short and to the point. That the SAR is regarded as repetitive by therapists suggests that it may be impeded by not just an insufficient number of unique phrases but also by the choice of phrases being repeated or the lack of variability in their delivery.

On occasion, the SAR's speech was observed to cause confusion or mild irritation in patient responses. For example, NAO's counting of exercise repetitions was observed to occasionally confuse patients when not in sync with their own perception of progress. Word pronunciation was also observed to be important. For example, while most patients visibly expressed satisfaction in the SAR referring to them by name, incorrect pronunciations were observed to evoke negative patient responses. One patient, for example, noted: "I would like the robot to say my name correctly." Such observations in patient reaction and performance, while highlighting clear need for improvement, also confirm the importance of robot speech in the SAR's design. Understanding how speech can be designed to best complement the roles of the SAR in such therapeutic contexts is an important area of future work.

Despite design decisions to optimise the robustness of NAO's built-in speech recognition (see Section 5.5.2), verbal interaction with the SAR remained problematic. Notably, recent studies have highlighted specific issues with the NAO platform's speech recognition [28] and natural language processing with children more generally [14]. Phase 2 session observations noted frequent falsenegative responses to simple words, such as "Go." This was observed especially with patients, but also with therapists. The provision of alternative modes of interaction allowed sessions to continue regardless. Notably, participants were observed to quickly discard verbal communication (typically after the first failed attempt) in favour of tactile button pressing to respond. Providing feedback to participants when speech was not recognised was observed to alleviate confusion and frustration, allowing participants to solve the situation themselves.

As noted, tactile button taps were observed to provide a reliable and preferred mode of interaction for both patients and therapists with the SAR. The inclusion of flashing LEDs marking the boundary of the head buttons was observed to reduce errors in precision and confusion caused by missed taps observed in Phase 1. In particular, the continued flashing of the LEDs until a tap 
(a) Time

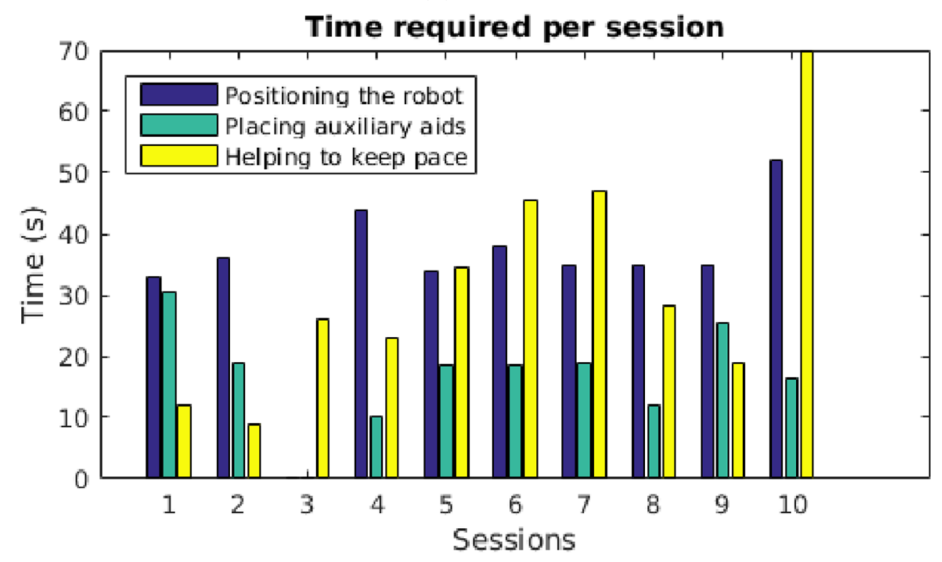

(b) Occurrences

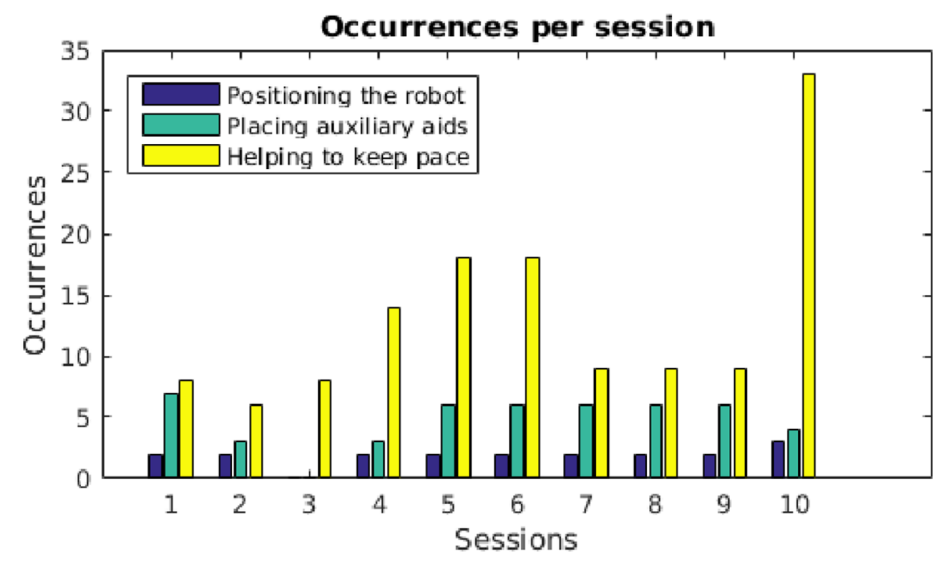

Fig. 10. Time required and number of occurrences per session for human-assisted actions.

was registered provided sufficient guidance to participants to make another attempt if required, further supporting the SAR's integration in the session and stand-alone operation.

6.4.4 Human-Assisted Activities. Figure 10(a) provides a coarse-level analysis of time costs associated with providing the SAR assistance over ten patient sessions in Phase 2. Figure 10(b) shows the corresponding number of occurrences of each activity for each session. It can be seen that assisting the robot to keep pace (via head touch) required less time to perform but occurred at significantly higher frequency than other human-assisted actions, scaling roughly with the number of activities to perform. While required often, keeping pace actions appeared to complement the general desire of patients to interact with the robot. If close enough to the robot, and able, patients performed the action themselves. Therapist feedback indicated that allowing patients to deliver assistance to NAO also appeared to increase their activity and engagement during the session.

Positioning the robot and placing auxiliary aids occurred less frequently than keeping pace actions but, as expected, required more session time to perform. However, therapists expressed no concern with this time cost (less than 1 minute). Thus, we consider the SAR's human-assistance needs to be within an acceptable limit. Notably, however, the exercise programs observed in the 
current study involve a relatively low number of human-assisted exercises. We note that other rehabilitation programs may include a more diverse range of exercises that may require more caregiver assistance.

Physiotherapists participated in the study without any prior training apart from being told that the SAR would ask for help from time to time. Therapists expressed willingness to provide assistance and, in general, demonstrated competence in handling the robot when required. A notable issue that was observed in sessions was the therapist attempting to perform tasks for the robot that it was capable of itself: in particular, placing the robot on its back for exercises. Therapists were not explicitly told that the SAR was capable of this itself and thus understandably intervened. Improvements to the SAR's instructions during sessions and more explicit statements of the SAR's capabilities during training should address this. In postsession interviews, no concerns were expressed about the impact of the assistance they were required to provide.

\subsection{Design Process Evaluation}

The SAR has engaged with over 40 unique patients across both phases of development. Within 23 months, we have progressed from exploration activities during informal visits to a base-level stand-alone therapeutic aid for rehabilitation, deployed in weekly clinical sessions. Phase 1 was necessarily unstructured, employing an in situ Wizard-of-Oz operation with therapists, patients, and parents. This is appropriate for busy clinical settings but could be complemented by formal requirement elicitation after a period of familiarisation.

Regular frequent in situ engagement with clinical stakeholders has been key to establishing trust and rapport. During Phase 1, therapist attitudes evolved from curious and unconvinced at the beginning to increasingly interested and engaged in the SAR's development and the design process. The design team now incorporates technical, physiotherapy, $\mathrm{CP}$ and psychology expertise. We argue that this in situ design process has been essential to the establishment of the SAR as a legitimate and viable therapeutic aid, which, in turn, has established clinical advocates for the SAR. This has been crucial to the recruitment of patients to participate in Phase 2 testing and to the long-term support of the project by the rehabilitation clinic.

Phase 1 established researchers' relationship with clinical staff and clinical concepts. The identification of a set of exercises that the robot was able to perform and the clinical knowledge of a group of patients that commonly are prescribed those exercises was key. Defining the target patient population and associated exercise set in consultation with therapists in Phase 1 allowed therapists to engage more directly with the design process by identifying appropriate patients to focus on and to recruit for Phase 2 testing. Notably, in more recent Phase 2 testing, the patient population has broadened to a larger population of children in rehabilitation, suggesting that the early focus on one patient cohort has not limited the scalability of the system to other patient groups.

We argue that the design of SARs for other health care applications may benefit from a similar design process of initial in situ exploration and stakeholder relationship building, leading then to the focussed development of a viable prototype for feasibility and technical capacity testing in Phase 2. We further advocate for a focus on discreet goals for the system, which, in our experience, allowed therapists to engage more readily with the process. Early Phase 1 attempts to present and demonstrate the general capabilities of the NAO system to therapists produced few outcomes, with no clear link to its practical implementation and therapeutic value.

The design process has provided therapists with direct access to the SAR system, allowing not only hands-on experience manipulating robot limbs but also experience with the software interface. While health professionals in general do not have the time (and perhaps interest) in this 
level of access, our experience has been that physiotherapists generally take up the opportunity, when offered, to explore the SAR's capabilities. This was observed to increase familiarity with the SAR's capabilities (and limitations) but, more importantly, provided an entry point for caregivers to directly contribute to the requirements analysis and design of the SAR. Whether the level of engagement we experienced is specific to physiotherapists or to the particular clinic is unclear. We argue, however, that providing frequent opportunities for stakeholders to engage with such novel and unfamiliar technology promotes transparency in the design process and a sense of ownership of the deployed system. This is a crucial feature of any design process that seeks to deploy SARs in a health care setting, where preconceptions and a lack of familiarity and trust of the technology (and the design process) risks impeding confidence and acceptance.

Certain limitations should be considered when designing in situ: regular on-site visitation requires a large time investment of a small, dedicated technical development team. Our approach promotes design and integration of an SAR into clinical practise but is not conducive to technical innovation by a small development team. Parallel lab-based development could be informed by, and feed into, Phase 2 prototype testing. Stakeholders' expectations must also be managed. While in situ development promotes design transparency, it also exposes delays and system failures directly to end-users. It is thus important to establish a common understanding of the constraints and limitations on both the system and the development cycle.

In situ design in a health care setting must carefully manage all of the above considerations within the context of a highly demanding and busy clinical environment. Technical developers must always concede to the needs of patients and therapists, which may often mean little progress is made in an individual session. High-frequency visitation can mitigate this, increasing opportunities for engagement with health care professionals and increasing their familiarity and acceptance of the technical development team.

\section{CONCLUSION}

We have presented our in situ design process for the development of a socially assistive robot for paediatric rehabilitation. Our two-phase process of exploration and development, embedded in the busy rehabilitation clinic of Melbourne's Royal Children's Hospital, has adapted a generalpurpose off-the-shelf social robot, NAO, as a stand-alone therapeutic aid deployed and leading weekly rehabilitation sessions with patients.

We have listed a set of roles and requirements for our system, derived from an initial exploratory phase in order to develop our first prototype. We have explained the design considerations in the current iterative development phase to satisfy the roles and requirements.

A deliberately conservative system has been deployed. While limited in capabilities, NAO's fasttracked deployment as a robust minimalist system is providing crucial patient engagement experience and insights into what is required for ongoing clinical deployment-in particular, a formal clinical evaluation of its therapeutic benefits. We argue that this approach has led to a system that not only meets minimum operational and therapeutic requirements for clinical deployment but also has clearly established priorities for further development as we prepare for formal clinical trials of the SAR for paediatric rehabilitation. Such outcomes offer insights into SAR design and development for other health care applications, particularly in busy clinic/hospital settings.

\section{ACKNOWLEDGMENTS}

We gratefully acknowledge the physiotherapists, patients and parents involved in this study. We also acknowledge The Brainary for initiating this project, and their ongoing general support of this study. 


\section{REFERENCES}

[1] Marina Umaschi Bers, Edith Ackermann, Justine Cassell, Beth Donegan, Joseph Gonzalez-Heydrich, David Ray DeMaso, Carol Strohecker, Sarah Lualdi, Dennis Bromley, and Judith Karlin. 1998. Interactive storytelling environments: Coping with cardiac illness at Boston's Children's Hospital. In Proceedings of the SIGCHI Conference on Human Factors in Computing Systems (CHI'98). ACM Press/Addison-Wesley Publishing Co., New York, NY, 603-610. DOI : http://dx.doi.org/10.1145/274644.274725

[2] B. Borovac, M. Gnjatović, S. Savić, M. Raković, and M. Nikolić. 2016. Human-like Robot MARKO in the Rehabilitation of Children with Cerebral Palsy. In New Trends in Medical and Service Robots: Assistive, Surgical and Educational Robotics, Hannes Bleuler, Mohamed Bouri, Francesco Mondata et al., eds. Springer International Publishing, Cham, Switzerland, 191-203. DOI : http://dx.doi.org/10.1007/978-3-319-23832-6_16

[3] Wolfram Burgard, Armin B. Cremers, Dieter Fox, Dirk Hähnel, Gerhard Lakemeyer, Dirk Schulz, Walter Steiner, and Sebastian Thrun. 1999. Experiences with an interactive museum tour-guide robot. Artificial Intelligence 114, 1-2, 3-55. DOI : http://dx.doi.org/10.1016/S0004-3702(99)00070-3

[4] L. V. Calderita, P. Bustos, C. Suárez Mejías, F. Fernández, and A. Bandera. 2013. THERAPIST: Towards an autonomous socially interactive robot for motor and neurorehabilitation therapies for children. In 7th International Conference on Pervasive Computing Technologies for Healthcare and Workshops. 374-377. DOI:http://dx.doi.org/10. 4108/icst.pervasivehealth.2013.252348

[5] Wan-Ling Chang, Selma Šabanović, and Lesa Huber. 2013. Situated analysis of interactions between cognitively impaired older adults and the therapeutic robot PARO. In Social Robotics. ICSR 2013, G. Herrmann, M. J. Pearson, A. Lenz, P. Bremner, A. Spiers, and U. Leonards (Eds.). Lecture Notes in Computer Science, vol. 8239. Springer, Cham.

[6] Vijay Chidambaram, Yueh-Hsuan Chiang, and Bilge Mutlu. 2012. Designing persuasive robots: How robots might persuade people using vocal and nonverbal cues. In Proceedings of the 7th Annual ACM/IEEE International Conference on Human-Robot Interaction (HRI'12). ACM, New York, NY, 293-300. DOI : http://dx.doi.org/10.1145/2157689.2157798

[7] Juan Fasola and Maja J. Matarić. 2013. A socially assistive robot exercise coach for the elderly. Fournal of Human-Robot Interaction 2, 2, 3-32. DOI : http://dx.doi.org/10.5898/JHRI.2.2.Fasola

[8] David Feil-Seifer and Maja J. Matarić. 2005. Defining socially assistive robotics. In 9th International Conference on Rehabilitation Robotics (ICORR'05). IEEE, 465-468. DOI : http://dx.doi.org/10.1109/ICORR.2005.1501143

[9] Marina Fridin and Mark Belokopytov. 2014. Robotics agent coacher for CP motor function (RAC CP fun). Robotica 32, 08, 1265-1279. DOI : http://dx.doi.org/10.1017/S026357471400174X

[10] Leonardo Giusti and Patrizia Marti. 2008. Robots as social mediators: A study 'in the wild.' Gerontechnology 7, 2, 113.

[11] Marcel Heerink, Ben Krose, Vanessa Evers, and Bob Wielinga. 2009. Measuring acceptance of an assistive social robot: A suggested toolkit. In RO-MAN 2009 - The 18th IEEE International Symposium on Robot and Human Interactive Communication. 528-533. DOI : http://dx.doi.org/10.1109/ROMAN.2009.5326320

[12] H. Hüttenrauch and K. S. Eklundh. 2002. Fetch-and-carry with CERO: Observations from a long-term user study with a service robot. In Proceedings of the 11th IEEE International Workshop on Robot and Human Interactive Communication. 158-163. DOI : http://dx.doi.org/10.1109/ROMAN.2002.1045615

[13] Helge Hüttenrauch, Elin Anna Topp, and Kerstin Severinson Eklundh. 2009. The art of gate-crashing: bringing HRI into users' homes. Interaction Studies 10, 3, 274-297. http://dx.doi.org/10.1075/is.10.3.02hut

[14] James Kennedy, Séverin Lemaignan, Caroline Montassier, Pauline Lavalade, Bahar Irfan, Fotios Papadopoulos, Emmanuel Senft, and Tony Belpaeme. 2017. Child speech recognition in human-robot interaction: Evaluations and recommendations. In Proceedings of the 2017 ACM/IEEE International Conference on Human-Robot Interaction (HRI'17). ACM, New York, NY, 82-90. DOI : http://dx.doi.org/10.1145/2909824.3020229

[15] Cory D. Kidd and Cynthia Breazeal. 2008. Robots at home: Understanding long-term human-robot interaction. In 2008 IEEE/RSf International Conference on Intelligent Robots and Systems. IEEE, 3230-3235. DOI : http://dx.doi.org/10. 1109/IROS.2008.4651113

[16] C. D. Kidd, W. Taggart, and S. Turkle. 2006. A sociable robot to encourage social interaction among the elderly. In Proceedings of the 2006 IEEE International Conference on Robotics and Automation (ICRA'06). 3972-3976. DOI : http://dx.doi.org/10.1109/ROBOT.2006.1642311

[17] Volodymyr Kozyavkin, Oleh Kachmar, and Iryna Ablikova. 2014. Humanoid social robots in the rehabilitation of children with cerebral palsy. In Proceedings of the 8th International Conference on Pervasive Computing Technologies for Healthcare (PervasiveHealth'14). ICST (Institute for Computer Sciences, Social-Informatics and Telecommunications Engineering), Brussels, Belgium, 430-431. DOI : http://dx.doi.org/10.4108/icst.pervasivehealth.2014.255323

[18] Tara E. Lambert, Lisa A. Harvey, Christos Avdalis, Lydia W. Chen, Sayanthinie Jeyalingam, Carin A. Pratt, Holly J. Tatum, Jocelyn L. Bowden, and Barbara R. Lucas. 2017. An app with remote support achieves better adherence to home exercise programs than paper handouts in people with musculoskeletal conditions: A randomised trial. fournal of Physiotherapy 63, 3, 161-167. DOI : http://dx.doi.org/10.1016/j.jphys.2017.05.015 
[19] Kwan Min Lee, Younbo Jung, Jaywoo Kim, and Sang Ryong Kim. 2006. Are physically embodied social agents better than disembodied social agents?: The effects of physical embodiment, tactile interaction, and people's loneliness in human-robot interaction. International fournal of Human-Computer Studies 64, 10, 962-973. DOI : http://dx.doi.org/10. 1016/j.ijhcs.2006.05.002

[20] Norjasweeen Abdul Malik, Hanafiah Yussof, Fazah Akhtar Hanapiah, Rabiatul Adawiah Abdul Rahman, and Husna Hassan Basri. 2015. Human-robot interaction for children with cerebral palsy: Reflection and suggestion for interactive scenario design. In 2015 IEEE International Symposium on Robotics and Intelligent Sensors (IEEE IRIS'15). Procedia Computer Science 76, Supplement C, 388-393. DOI : http://dx.doi.org/10.1016/j.procs.2015.12.315

[21] Felip Martí Carrillo, Jo Butchart, Sarah Knight, Adam Scheinberg, Lisa Wise, Leon Sterling, and Chris McCarthy. 2017. In-situ design and development of a socially assistive robot for paediatric rehabilitation. In Proceedings of the Companion of the 2017 ACM/IEEE International Conference on Human-Robot Interaction (HRI'17). ACM, New York, NY, 199-200. DOI : http://dx.doi.org/10.1145/3029798.3038382

[22] Felip Martí Carrillo, Jo Butchart, Sarah Knight, Adam Scheinberg, Lisa Wise, Leon Sterling, and Chris McCarthy. 2016. Help me help you: A human-assisted social robot in paediatric rehabilitation. In Proceedings of the 28th Australian Conference on Computer-Human Interaction (OzCHI'16). ACM, New York, NY, 659-661. DOI : http://dx.doi.org/10.1145/ 3010915.3011858

[23] Chris McCarthy, Jo Butchart, Michael George, Dee Kerr, Hugh Kingsley, Adam M. Scheinberg, and Leon Sterling. 2015. Robots in rehab: Towards socially assistive robots for paediatric rehabilitation. In Proceedings of the Annual Meeting of the Australian Special Interest Group for Computer Human Interaction (OzCHI'15). ACM, New York, NY, 39-43. DOI : http://dx.doi.org/10.1145/2838739.2838791

[24] Thomas McDonald, Graciela Couchonnal, and Theresa Early. 1996. The impact of major events on the lives of family caregivers of children with disabilities. Families in Society: The fournal of Contemporary Social Services 77, 8, 502-514. DOI : http://dx.doi.org/10.1606/1044-3894.960

[25] Marek P. Michalowski, Selma Šabanović, Carl DiSalvo, Didac Busquets, Laura M. Hiatt, Nik A. Melchior, and Reid Simmons. 2007. Socially distributed perception: GRACE plays social tag at AAAI 2005. Autonomous Robots 22, 4, 385-397. DOI : http://dx.doi.org/10.1007/s10514-006-9015-6

[26] Wendy Moyle, Marie Cooke, Elizabeth Beattie, Cindy Jones, Barbara Klein, Glenda Cook, Chrystal Gray, and others. 2013. Exploring the effect of companion robots on emotional expression in older adults with dementia: A pilot randomized controlled trial. Journal of Gerontological Nursing 39, 5, 46-53. https://dx.doi.org/10.3928/ 00989134-20130313-03

[27] Bilge Mutlu and Jodi Forlizzi. 2008. Robots in organizations: The role of workflow, social, and environmental factors in human-robot interaction. In Proceedings of the 3rd ACM/IEEE International Conference on Human Robot Interaction (HRI'08). ACM, New York, NY, 287-294. DOI : http://dx.doi.org/10.1145/1349822.1349860

[28] Hannah R. M. Pelikan and Mathias Broth. 2016. Why that NAO?: How humans adapt to a conventional humanoid robot in taking turns-at-talk. In Proceedings of the 2016 CHI Conference on Human Factors in Computing Systems (CHI'16). ACM, New York, NY, 4921-4932. DOI : http://dx.doi.org/10.1145/2858036.2858478

[29] Catherine Plaisant, Allison Druin, Corinna Lathan, Kapil Dakhane, Kris Edwards, Jack Maxwell Vice, and Jaime Montemayor. 2000. A storytelling robot for pediatric rehabilitation. In Proceedings of the F4th International ACM Conference on Assistive Technologies (Assets'00). ACM, New York, NY, 50-55. DOI : http://dx.doi.org/10.1145/354324. 354338

[30] K. M. Plant and M. R. Sanders. 2007. Predictors of care-giver stress in families of preschool-aged children with developmental disabilities. fournal of Intellectual Disability Research 51, 2, 109-124. DOI: http://dx.doi.org/10.1111/j.1365-2788. 2006.00829.x

[31] E. Pot, J. Monceaux, R. Gelin, and B. Maisonnier. 2009. Choregraphe: A graphical tool for humanoid robot programming. In RO-MAN 2009 - The 18th IEEE International Symposium on Robot and Human Interactive Communication. 46-51. DOI : http://dx.doi.org/10.1109/ROMAN.2009.5326209

[32] J. Pripfl, T. Körtner, D. Batko-Klein, D. Hebesberger, M. Weninger, C. Gisinger, S. Frennert, H. Eftring, M. Antona, I. Adami, A. Weiss, M. Bajones, and M. Vincze. 2016. Results of a real world trial with a mobile social service robot for older adults. In 11th ACM/IEEE International Conference on Human-Robot Interaction (HRI). 497-498. DOI : http://dx.doi.org/10.1109/HRI.2016.7451824

[33] Morgan Quigley, Ken Conley, Brian Gerkey, Josh Faust, Tully Foote, Jeremy Leibs, Rob Wheeler, and Andrew Y. Ng. 2009. ROS: An open-source robot operating system. In ICRA Workshop on Open Source Software. 5.

[34] Parminder Raina, Maureen O’Donnell, Peter Rosenbaum, Jamie Brehaut, Stephen D. Walter, Dianne Russell, Marilyn Swinton, Bin Zhu, and Ellen Wood. 2005. The health and well-being of caregivers of children with cerebral palsy. Pediatrics 115, 6, e626-e636. DOI : http://dx.doi.org/10.1542/peds.2004-1689

[35] Ben Robins, Kerstin Dautenhahn, R. Te Boekhorst, and Aude Billard. 2005. Robotic assistants in therapy and education of children with autism: Can a small humanoid robot help encourage social interaction skills? Universal Access in the Information Society 4, 2, 105-120. DOI : http://dx.doi.org/10.1007/s10209-005-0116-3 
[36] Yvonne Rogers. 2011. Interaction design gone wild: Striving for wild theory. Interactions 18, 4, 58-62. DOI : http://dx. doi.org/10.1145/1978822.1978834

[37] C. Suárez Mejías, C. Echevarría, P. Nuñez, L. Manso, P. Bustos, S. Leal, and C. Parra. 2013. Ursus: A Robotic Assistant for Training of Children with Motor Impairments. Springer, Berlin, 249-253. DOI: http://dx.doi.org/10.1007/ 978-3-642-34546-3_39

[38] Pam Thomason and H. Kerr Graham. 2013. Rehabilitation of children with cerebral palsy after single-event multilevel surgery. In Rehabilitation in Movement Disorders, R. Iansek and M. Morris (Eds.). Cambridge: Cambridge University Press, 203-216. DOI : https://doi.org/10.1017/CBO9781139012942.019

[39] Pam Thomason, Paulo Selber, and H. Kerr Graham. 2013. Single event multilevel surgery in children with bilateral spastic cerebral palsy: A 5 year prospective cohort study. Gait \& Posture 37, 1, 23-28. DOI: http://dx.doi.org/10.1016/ j.gaitpost.2012.05.022

[40] S. Thrun, M. Beetz, M. Bennewitz, W. Burgard, A. B. Cremers, F. Dellaert, D. Fox, D. Hiähnel, C. Rosenberg, N. Roy, J. Schulte, and D. Schulz. 2000. Probabilistic algorithms and the interactive museum tour-guide robot Minerva. The International fournal of Robotics Research 19, 11, 972-999. DOI : http://dx.doi.org/10.1177/02783640022067922

[41] Selma Šabanović, Sarah M. Reeder, and Bobak Kechavarzi. 2014. Designing robots in the wild: In situ prototype evaluation for a break management robot. fournal of Human-Robot Interaction 3, 1, 70-88. DOI : http://dx.doi.org/10. 5898/JHRI.3.1.Sabanovic.

[42] K. Wada, T. Shibata, T. Saito, K. Sakamoto, and K. Tanie. 2005. Psychological and social effects of one year robot assisted activity on elderly people at a health service facility for the aged. In Proceedings of the 2005 IEEE International Conference on Robotics and Automation. 2785-2790. DOI : http://dx.doi.org/10.1109/ROBOT.2005.1570535

[43] K. Wada, T. Shibata, T. Saito, and K. Tanie. 2004. Effects of robot-assisted activity for elderly people and nurses at a day service center. Proceedings IEEE 92, 11, 1780-1788. DOI : http://dx.doi.org/10.1109/JPROC.2004.835378

[44] Eric Wade, Avinash Rao Parnandi, and Maja J. Matarić. 2011. Using socially assistive robotics to augment motor task performance in individuals post-stroke. In 2011 IEEE/RSf International Conference on Intelligent Robots and Systems. IEEE, 2403-2408. DOI : http://dx.doi.org/10.1109/IROS.2011.6095107

[45] J. Wainer, D. J. Feil-Seifer, D. A. Shell, and M. J. Matarić. 2007. Embodiment and human-robot interaction: A task-based perspective. In RO-MAN 2007 - The 16th IEEE International Symposium on Robot and Human Interactive Communication. 872-877. DOI : http://dx.doi.org/10.1109/ROMAN.2007.4415207

Received November 2017; revised February 2018; accepted March 2018 